\title{
THE ABUNDANCES OF NEUTRON-CAPTURE SPECIES IN THE VERY METAL-POOR GLOBULAR CLUSTER M15: A UNIFORM ANALYSIS OF RED GIANT BRANCH AND RED HORIZONTAL BRANCH STARS
}

\author{
Jennifer S. Sobeck ${ }^{1}$, Robert P. Kraft ${ }^{2}$, Christopher Sneden ${ }^{3}$, George W. Preston ${ }^{4}$, John J. Cowan ${ }^{5}$, Graeme H. Smith ${ }^{2}$, \\ Ian B. Thompson ${ }^{4}$, Stephen A. Shectman $^{4}$, And Gregory S. Burley ${ }^{4}$ \\ ${ }^{1}$ Department of Astronomy and Astrophysics, University of Chicago, 5640 South Ellis Avenue, Chicago, IL 60637, USA; jsobeck@ uchicago.edu \\ ${ }^{2}$ Department of Astronomy and Astrophysics, University of California-Santa Cruz, Santa Cruz, CA 95064, USA; kraft@ucolick.org, graeme@ucolick.org \\ ${ }^{3}$ Department of Astronomy, University of Texas-Austin, 1 University Station C1400, Austin, TX 78712, USA; chris@ verdi.as.utexas.edu \\ ${ }^{4}$ The Observatories of the Carnegie Institution of Washington, 813 Santa Barbara Street, Pasadena, CA 91101, USA; gwp@ obs.carnegiescience.edu, \\ ian@obs.carnegiescience.edu, sshectman@obs.carnegiescience.edu, burley@obs.carnegiescience.edu \\ ${ }^{5}$ Homer L. Dodge Department of Physics and Astronomy, University of Oklahoma, Norman, OK 73019, USA; cowan@nhn.ou.edu \\ Received 2010 November 23; accepted 2011 February 22; published 2011 April 20
}

\begin{abstract}
The globular cluster M15 is unique in its display of star-to-star variations in the neutron-capture elements. Comprehensive abundance surveys have been previously conducted for handfuls of M15 red giant branch (RGB) and red horizontal branch (RHB) stars. No attempt has been made to perform a single, self-consistent analysis of these stars, which exhibit a wide range in atmospheric parameters. In the current effort, a new comparative abundance derivation is presented for three RGB and six RHB members of the cluster. The analysis employs an updated version of the line transfer code MOOG, which now appropriately treats coherent, isotropic scattering. The apparent discrepancy in the previously reported values for the metallicity of M15 RGB and RHB stars is addressed and a resolute disparity of $\Delta(\mathrm{RHB}-\mathrm{RGB}) \approx 0.1 \mathrm{dex}$ in the iron abundance was found. The anti-correlative behavior of the light neutron-capture elements $(\mathrm{Sr}, \mathrm{Y}, \mathrm{Zr}$ ) is clearly demonstrated with both $\mathrm{Ba}$ and $\mathrm{Eu}$, standard markers of the $s$ - and $r$-process, respectively. No conclusive detection of $\mathrm{Pb}$ was made in the RGB targets. Consequently for the M15 cluster, this suggests that the main component of the $s$-process has made a negligible contribution to those elements normally dominated by this process in solar system material. Additionally for the M15 sample, a large Eu abundance spread is confirmed, which is comparable to that of the halo field at the same metallicity. These abundance results are considered in the discussion of the chemical inhomogeneity and nucleosynthetic history of M15.
\end{abstract}

Key words: globular clusters: individual (M15) - radiative transfer - stars: abundances - stars: horizontal-branch - stars: late-type

Online-only material: color figures, machine-readable and VO tables

\section{INTRODUCTION}

Detections of multiple main sequences and giant branches in globular clusters (GCs; e.g., $\omega$ Cen, NGC 2808, and NGC 1851; Bedin et al. 2004; Piotto et al. 2007; Han et al. 2009) have challenged the notion that these objects are uniformly mono-metallic stellar systems of unique age. In addition to the metallicity variations observed in certain clusters, the anomalous abundance behaviors of globulars include star-to-star scatter of light element $[\mathrm{el} / \mathrm{Fe}]$ ratios (for $\mathrm{C}, \mathrm{N}, \mathrm{O}, \mathrm{Na}, \mathrm{Mg}$, and $\mathrm{Al}$ ) in both main sequence and giant stars (this is in contrast to the abundance trends of halo field stars; e.g., Carretta et al. 2009a; Gratton et al. 2004). ${ }^{6}$ These departures in the relative abundances (found in stars of different evolutionary stages) imply that there are multiple stellar generations present within the $\mathrm{GC}$ and that an initial generation may have contributed to the intracluster medium (ICM). It is possible that three sources are responsible for the aggregate chemical makeup of a GC: a primordial source that generates the initial composition of the protocluster cloud, a pollution source that deposits material into the ICM from highly evolved asymptotic branch stars, and a mixing source that is independent of the other two and the result of stellar evolution processes. Further discussion of these sce-

\footnotetext{
6 We adopt the standard spectroscopic notation (Helfer et al. 1959) that for elements $\mathrm{A}$ and $\mathrm{B},[\mathrm{A} / \mathrm{B}] \equiv \log _{10}\left(N_{\mathrm{A}} / N_{\mathrm{B}}\right)_{\star}-\log _{10}\left(N_{\mathrm{A}} / N_{\mathrm{B}}\right)_{\odot}$. We also employ the definition $\log \epsilon(\mathrm{A}) \equiv \log _{10}\left(N_{\mathrm{A}} / N_{\mathrm{H}}\right)+12.0$.
}

narios may be found in, e.g., Bekki et al. (2007) and Carretta et al. (2009b).

On the other hand, in the vast majority of GCs, minimal scatter in the element abundance ratios with $Z>20$ has been observed. The abundances for the neutron $(n$-) capture elements europium and barium have been measured in several GCs, and only in a few exceptional cases have significantly large intracluster differences in these values been seen (e.g., M22; Marino et al. 2009). The predominant mechanism of $\mathrm{Eu}$ manufacture is the rapid $n$-capture process ( $r$-process), whereas the primary nucleosynthetic channel for $\mathrm{Ba}$ is slow $n$-capture (s-process; additional information pertaining to these production mechanisms may be found in, e.g., Sneden et al. $2008)$. Consequently, the abundance ratio of $[\mathrm{Eu} / \mathrm{Ba}]$ is used to demonstrate the relative prevalence of the $r$ - or $s$-process in individual stars. In GCs with a metallicity of $[\mathrm{Fe} / \mathrm{H}] \lesssim-1$, a general enhancement of $[\mathrm{Eu} / \mathrm{Ba}] \sim+0.4$ to +0.6 dex is detected, which indicates that $n$-capture element production has been dominated by the $r$-process (Gratton et al. 2004, and references therein). This in turn is suggestive of explosive nucleosynthetic input from very massive stars.

The very metal-deficient GC M15 (NGC 7078; $[\mathrm{Fe} / \mathrm{H}]$ -2.3 ) has been subject to several abundance investigations including the recent study by Carretta et al. (2009a). They employed both medium-resolution and high-resolution spectra of over 80 red giant stars to precisely determine the metallicity 
of this cluster: $\langle[\mathrm{Fe} / \mathrm{H}]\rangle=-2.314 \pm 0.007$. Additionally, they detected variations in the light element abundances ( $\mathrm{Na}$ and $\mathrm{O}$ ) for stars along the entirety of the red giant branch (RGB). Prior studies of M15 have also observed large scatter in the relative $\mathrm{Ba}$ and $\mathrm{Eu}$ (intracluster) abundances. With the spectra of 17 RGB stars, Sneden et al. (1997) found a factor of three spread in both ratios: $\langle[\mathrm{Ba} / \mathrm{Fe}]\rangle=0.07 ; \sigma=0.18$ and $\langle[\mathrm{Eu} / \mathrm{Fe}]\rangle=0.49 ; \sigma=0.20 .^{7}$ They were able to exclude measurement error as the source for the scatter and determined that the variations were correlated: $\langle[\mathrm{Eu} / \mathrm{Ba}]\rangle=0.41 ; \sigma=$ 0.11 . In a follow-up study of 31 M15 giants by Sneden et al. (2000b), the scatter in the relative Ba abundance was confirmed: $\langle[\mathrm{Ba} / \mathrm{Fe}]\rangle=0.12 ; \sigma=0.21$ (limitations in the spectral coverage did not permit a corresponding analysis of $\mathrm{Eu}$ ).

The majority of M15 high-resolution abundance analyses have employed yellow-red visible spectra to maximize the signal-to-noise ratio $(\mathrm{S} / \mathrm{N}$; stellar flux levels are relatively high for RGB targets in this region). In order to precisely derive the neutron-capture abundance distribution in M15, Sneden et al. (2000a) re-observed three tip giants in the blue visible wavelength regime (which contains numerous $n$-capture spectral transitions). The abundance determinations of eight $n$-capture species (Ba, La, Ce, Nd, Sm, Eu, Gd, and Dy) were performed and large star-to-star scatter in the all of the $[\mathrm{El} / \mathrm{Fe}]$ ratios was measured. They also found that the three stars exhibited a scaled solar system $r$-process abundance pattern. Additional verification of these abundance results was done by Otsuki et al. (2006) in an analysis of six M15 RGB stars (the two studies had one star in common, K462). Consistent with Sneden et al. (2000a), they detected significant variation in the $[\mathrm{Eu} / \mathrm{Fe}]$, $[\mathrm{La} / \mathrm{Fe}]$, and $[\mathrm{Ba} / \mathrm{Fe}]$ ratios. Furthermore, Otsuki et al. found that the ratios of $[(\mathrm{Y}, \mathrm{Zr}) / \mathrm{Eu}]$ show distinct anti-correlations with the Eu abundance. Finally employing an alternate stellar sample, Preston et al. (2006) examined six red horizontal branch (RHB) stars of M15. ${ }^{8}$ For the elements $\mathrm{Sr}, \mathrm{Y}, \mathrm{Zr}, \mathrm{Ba}$, and Eu, a large (star-to-star) spread in the abundances was measured. In essence, all of these investigations have observed considerable chemical inhomogeneity in the $n$-capture elements of the GC M15.

Two issues are brought to light by the M15 abundance data: the timescale and efficiency of mixing in the protocluster environment and the nucleosynthetic mechanism(s) responsible for $n$-capture element manufacture. In this GC, large abundance variations are seen in the two stellar evolutionary classes as well as in both the light and heavy neutron-capture species. There is a definitive enhancement of $r$-process elements found in some stars of M15 (e.g., K462), yet not exhibited in others (e.g., B584). Taking into consideration the entirety of the M15 n-capture results, these data hint at the existence of a nucleosynthetic mechanism different from the classical $r$ - and $s$-processes. Evidence of such a scenario (with multiple production pathways) also may be found in halo field stars of analogous metallicity such as CS 22892-052 (Sneden et al. 2003) and HD 122563 (Honda et al. 2006), which have displayed similar abundance variations. Indeed, several models have advanced the notion of more than one $r$-process formation scenario (e.g., Wasserburg \& Qian 2000; Qian \& Wasserburg 2002; Thielemann et al. 2001; Kratz et al. 2007).

\footnotetext{
7 The anomalously nitrogen-enriched star K969 is omitted; see Appendix A of Sneden et al. (1997).

8 The papers from Sneden et al. (1997, 2000a, 2000b) and Preston et al. (2006) are from collaborators affiliated with institutions in both California and Texas. Hereafter, these papers and other associated publications will be referred to as CTG.
}

To further understand the implications of the M15 results, the spectra from the three RGB stars of Sneden et al. (2000a) and the six RHB stars of Preston et al. (2006) are re-analyzed. A single consistent methodology for the analysis is employed and an expansive set of recently determined oscillator strengths is utilized (e.g., Lawler et al. 2009; Sneden et al. 2009, and references therein). As these stars are re-examined, the relative invariance of the abundance distributions will be ascertained for $r$ - and $s$-process species. In consideration of the M15 investigations cited above, a few data anomalies have come to light. The two main issues to be resolved include: large discrepancies in the $\log \epsilon(E l)$ values between the studies of Sneden et al. (2000a) and Otsuki et al. (2006); and the significant disparity in the derived metallicity for the M15 cluster between Preston et al. $\left(2006,\langle[\mathrm{Fe} / \mathrm{H}]\rangle_{\mathrm{RHB}}=-2.63\right)$ and the canonically accepted value of $\langle[\mathrm{Fe} / \mathrm{H}]\rangle=-2.3$ (e.g., Carretta et al. 2009a; Sneden et al. 2000a). It is suggested that these differences are mostly due to selection of atomic data, model atmosphere, and treatment of scattering.

\section{OBSERVATIONAL DATA}

For the three RGB stars of the M15 cluster, the re-analysis of two sets of high-resolution spectra was performed: the first from Sneden et al. (1997) with approximate wavelength coverage region of $5400 \AA \lesssim \lambda \lesssim 6800 \AA$ and the second from Sneden et al. (2000a) with a wavelength domain of $3600 \AA \lesssim \lambda \lesssim$ $5200 \AA$. All spectral observations were acquired with the High Resolution Echelle Spectrometer (Vogt et al. 1994) at the Keck I $10.0 \mathrm{~m}$ telescope (with a spectral resolving power of $R \equiv \lambda / \Delta \lambda \simeq$ $45,000)$. The $\mathrm{S} / \mathrm{N}$ range of the data varied from $30 \lesssim \mathrm{S} / \mathrm{N} \lesssim 150$ for the shorter wavelength spectra to $100 \lesssim \mathrm{S} / \mathrm{N} \lesssim 150$ for the longer wavelength spectra (the $\mathrm{S} / \mathrm{N}$ value generally increased with wavelength). The three giants, K341, K462, and K583, ${ }^{9}$ were selected from the larger stellar sample of Sneden et al. (1997) due to relative brightness, rough equivalence of model atmospheric parameters, and extreme spread in associated $\mathrm{Ba}$ and Eu abundances.

Re-examination of the high-resolution spectra of six RHB stars from the study of Preston et al. (2006) was also done. The observations were taken at the Magellan Clay $6.5 \mathrm{~m}$ telescope of the Las Campanas Observatory with the Magellan Inamori Kyocera Echelle spectrograph (Bernstein et al. 2003). The data had a resolution of $R \simeq 40,000$ and the $S / N$ values ranged from $\mathrm{S} / \mathrm{N} \sim 25$ at $3600 \AA$ to $\mathrm{S} / \mathrm{N} \sim 120$ at $7200 \AA$ (note that almost complete spectral coverage was obtained in the region $3600 \AA \lesssim \lambda \lesssim 7200 \AA$ ). The six RHB targets were chosen from the photometric catalog of Buonanno et al. (1983) and accordingly signified as B009, B028, B224, B262, B412, and B584. It should be pointed out that these stars have significantly lower temperatures than other HB members (and thus, match up favorably with the RGB).

Figure 1 features the color-magnitude diagram (CMD) for the M15 GC with a plot of the $V$ versus $(V-K)$ magnitudes. The $V$ magnitudes for the RGB stars are taken from the preliminary results of K. M. Cudworth (2011, private communication) and verified against the data from Cudworth (1976). Alternatively, the RHB $V$ magnitude values are obtained from Buonanno et al. (1983). The $K$ magnitudes for all M15 targets are taken from the Two Micron All Sky Survey (2MASS; Skrutskie et al. 2006). Cluster members with both $B$ and $V$ measurements from Buonanno et al. are displayed in the plot (denoted by the black

\footnotetext{
9 The Kustner (1921) designations are employed throughout the text.
} 


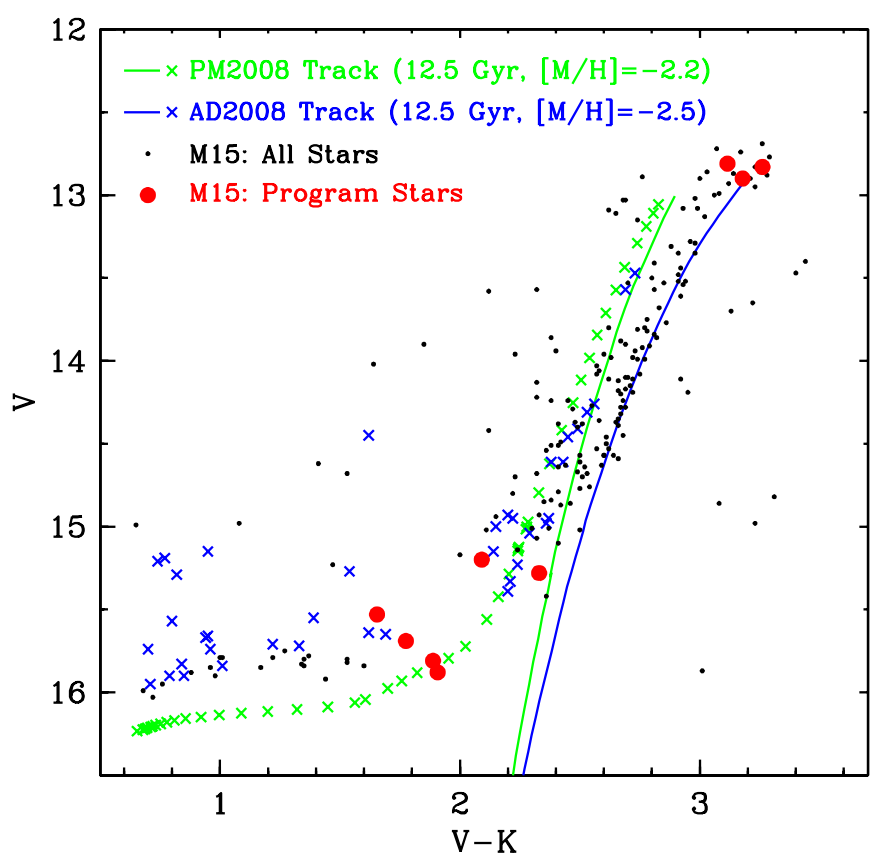

Figure 1. CMD for the globular cluster M15. For cluster members (indicated by small black dots), $V$ magnitudes are taken Buonanno et al. (1983) while the $K$ magnitudes are obtained from the 2MASS database (Skrutskie et al. 2006). Note that stars with both $B$ and $V$ magnitudes from Buonanno et al. are shown in the plot. The program stars are signified by large, red circles. Also displayed are the isochrone data from both Marigo et al. (2008; labeled PM2008; shown in green) and Dotter et al. (2008; labeled AD2008; shown in blue).

(A color version of this figure is available in the online journal.)

dots) and the stars of the current study are indicated by large, red circles. Note that the identifications of RGB and RHB members are based upon stellar atmospheric parameters as well as the findings from Sneden et al. (1997) and Preston et al. (2006; please consult those references for additional details). Also in Figure 1, two isochrone determinations are overlaid upon the photometric data: Marigo et al. (2008; with the age parameter set to $12.5 \mathrm{Gyr}$ and a metallicity of $[\mathrm{M} / \mathrm{H}]=-2.2$, shown in green) and Dotter et al. (2008; with the age parameter set to $12.5 \mathrm{Gyr}$ and a metallicity of $[\mathrm{M} / \mathrm{H}]=-2.5$, shown in blue). These are the best-fit isochrones to the general characteristics ascribed to M15 and no preference is given to either source.

Additional observational details of the aforementioned data samples may be found in the original Sneden et al. (1997, 2000a) and Preston et al. (2006) publications. These papers also contain descriptions of the data reduction procedures, in which standard IRAF ${ }^{10}$ tasks were used for extraction of multi-order wavelength-calibrated spectra from the raw data frames, and specialized software (SPECTRE; Fitzpatrick \& Sneden 1987) was employed for continuum normalization and cosmic ray elimination.

Figure 2 features a comparison of the spectra of all M15 targets. Displayed in this plot is a small wavelength interval 4121-4133 $\AA$, which highlights the important $n$-capture transitions La II at $4123.22 \AA$ and Eu II at $4129.72 \AA$. The spectra are arranged in decreasing $T_{\text {eff }}$ from top to bottom of the figure. As shown, the combined effects of $T_{\text {eff }}$ and $\log g$ influence the apparent line strength, and accordingly, transitions which

\footnotetext{
10 IRAF is distributed by the National Optical Astronomy Observatory, which is operated by the Association of Universities for Research in Astronomy, Inc., under cooperative agreement with the National Science Foundation.
}

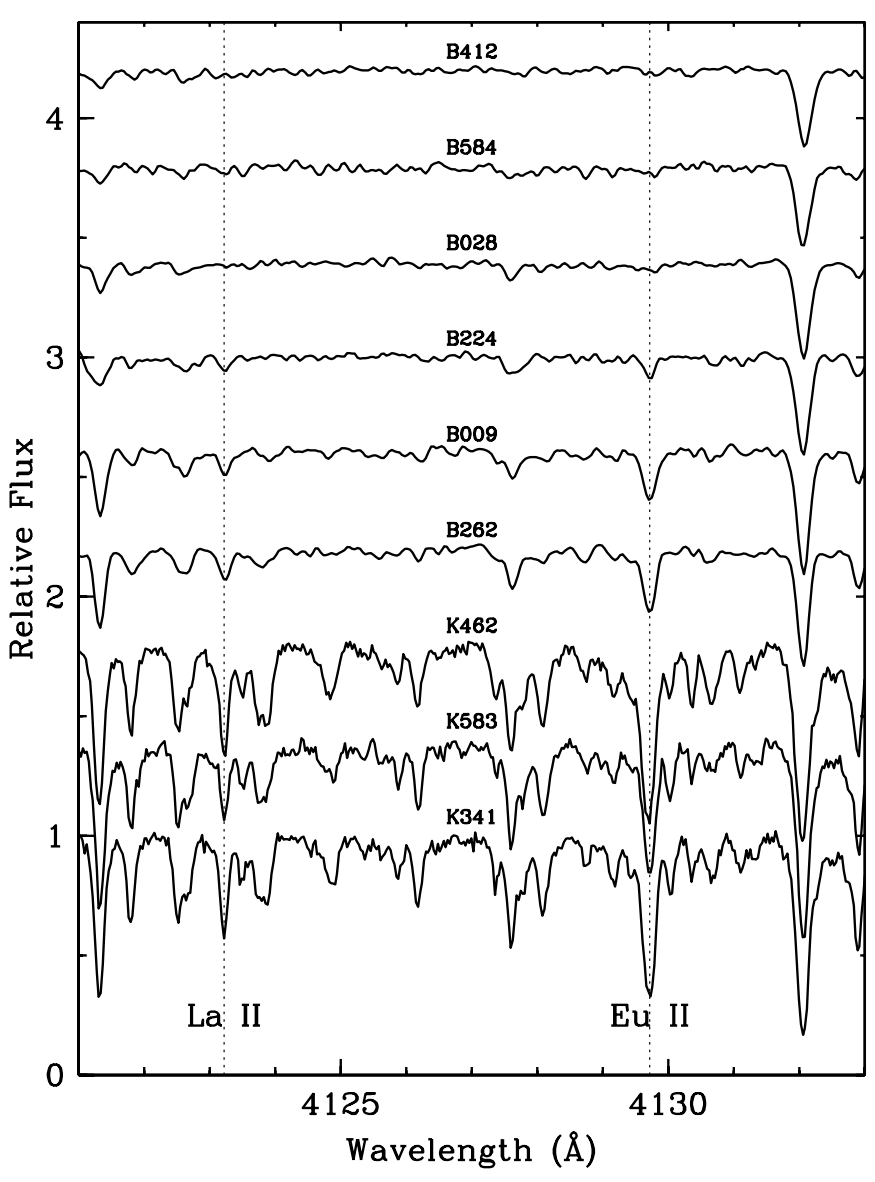

Figure 2. Small wavelength region shown for all program stars. For the purposes of display, the relative fluxes of target stars (other than K341) have been shifted by additive constants. Vertical dotted lines denote the spectral features of La II at a wavelength of $4123.22 \AA$ and of Eu II at $4129.72 \AA$. Note that these transitions appear to be more pronounced in the spectra of the lower temperature RGB targets.

are saturated in the RGB spectra completely disappear in the warmer RHB spectra.

\section{METHODOLOGY AND MODEL DETERMINATION}

Several measures were implemented in order to improve and extend the efforts of Sneden et al. (1997, 2000a) and Preston et al. (2006). First, the modification of the line analysis program MOOG was performed to accurately ascertain the relative contributions to the continuum opacity (especially necessary for the bluer wavelength regions and the cool, metal-poor RGB targets). Second, the employment of an alternative grid of models was done to obtain an internally consistent set of stellar atmospheric parameters for the total M15 sample. Third, the utilization of the most up-to-date experimentally and semiempirically derived transition probability data was done to determine the abundances from multiple species.

\subsection{Atomic Data}

Special effort was made to employ the most recent laboratory measurements of oscillator strengths. When applicable, the inclusion of hyperfine and isotopic structure was done for the derivation of abundances. Tables 2 and 3 list the various literature sources for the transition probability data. Some species deserve special comment. The Fe transition probability values are taken from the critical compilation of Fuhr \& 
Wiese (2006; note for neutral Fe, the authors heavily weigh the laboratory data from O'Brian et al. 1991). No up-to-date laboratory work has been done for $\mathrm{Sr}$, and so, the adopted $g f$-values are from the semi-empirical study by Brage et al. (1998; these values are in good agreement with those derived empirically by Gratton \& Sneden 1994). Similarly, the most recent laboratory effort for Y was by Hannaford et al. (1982). Yet these transition probabilities appear to be robust, yielding small line-to-line scatter.

A particular emphasis of the current work is the $n$-capture element abundances, for which a wealth of new transition probability data have become recently available. Correspondingly, the extensive sets of rare Earth $g f$-values from the Wisconsin Atomic Physics Group were adopted (Sneden et al. 2009; Lawler et al. 2009, and references therein). These data when applied to the solar spectrum yield photospheric abundances that are in excellent agreement with meteoritic abundances. For neutron-capture elements not studied by the Wisconsin group (which include $\mathrm{Ba}, \mathrm{Pr}, \mathrm{Yb}, \mathrm{Os}$, Ir, and $\mathrm{Th}$ ), alternate literature references were employed (and these are accordingly given in the two aforementioned tables).

\subsection{Consideration of Isotropic, Coherent Scattering}

In the original version of the line transfer code MOOG (Sneden 1973), local thermodynamic equilibrium (LTE) was assumed and hence, scattering was treated as pure absorption. Accordingly, the source function, $S_{\lambda}$, was set equal to the Planck function, $B_{\lambda}(T)$, which is an adequate assumption for metal-rich stars in all wavelength regions. However, for the extremely metal-deficient, cool M15 giants, the dominant source of opacity switches from $\mathrm{H}^{-}$BF to Rayleigh scattering in the blue visible and ultraviolet wavelength domain $(\lambda \lesssim 4500 \AA)$. It was then necessary to modify the MOOG program as the LTE approximation was no longer sufficient (this has also been remarked upon by other abundance surveys, e.g., Johnson 2002; Cayrel et al. 2004).

The classical assumptions of one-dimensionality and planeparallel geometry continue to be employed in the code. Now with the inclusion of isotropic, coherent scattering, the framework for solution of the radiative transfer equation (RTE) shifts from an initial value to a boundary value problem. The source function then assumes the form ${ }^{11}$ of $S=(1-\epsilon) J+\epsilon B$ and the description of line transfer becomes an integro-differential equation. The chosen methodology for the solution of the RTE (and the determination of mean intensity) is the approach of short characteristics that incorporates aspects of an accelerated convergence scheme. In essence, the short characteristics technique employs a tensor product grid in which the interpolation of intensity values occurs at selected grid points. The prescription generally followed was that from Koesterke et al. (2002 and references therein). The Appendix provides more detail with regard to the MOOG program alterations.

Prior to these modifications, for a low-temperature and lowmetallicity star (e.g., an RGB target), the ultraviolet and blue visible spectral transitions reported aberrantly high abundances in comparison to those abundances found from redder lines. With the implementation of the revised code, better line-toline agreement is found and accordingly, the majority of the abundance trend with wavelength is eliminated for these types

\footnotetext{
11 To re-state, the equation terms are defined as follows: $S$ is the source function, $\epsilon$ is the thermal coupling parameter, $J$ is the mean intensity, and $B$ is the Planck function.
}

of stars. Note for the RHB targets, minimal changes are seen in abundances with the employment of the modified MOOG program (as the dominant source of opacity for these relatively warm stars is always $\mathrm{H}^{-} \mathrm{BF}$ over the spectral region of interest).

\subsection{Atmospheric Parameter Determination}

To obtain preliminary estimates of $T_{\text {eff }}$ and $\log g$ for the M15 stars, photometric data from the aforementioned sources (K. M. Cudworth 2011, private communication; Buonanno et al. 1983; 2MASS) were employed as well as those data from Yanny et al. 1994. To transform the color, the color- $T_{\text {eff }}$ relations of Alonso et al. (1999) were used in conjunction with the distance modulus $\left((m-M)_{0}=15.25\right)$ and reddening $(E(B-V)=$ 0.10) determinations from Kraft \& Ivans (2003). Note that an additional intrinsic uncertainty of about 0.1 dex in $\log g$ remains among luminous RGB stars owing to stochastic mass loss of order 0.1 dex. Consequently, initial masses of $0.8 M_{\odot}$ and $0.6 M_{\odot}$ were assumed for RGB and RHB stars, respectively. The photometric $V$ and $(V-K)$ values as well as the photometrically and spectroscopically derived stellar atmospheric parameters are collected in Table 1.

With the use of the spectroscopic data analysis program SPECTRE (Fitzpatrick \& Sneden 1987), the equivalents widths (EWs) of transitions from the elements TiI/II, CrI/II, and Fe I/II were measured in the wavelength range 3800-6850. The preliminary $T_{\text {eff }}$ values were adjusted to achieve zero slope in plots of Fe abundance $\left(\log \left(\epsilon_{\mathrm{Fe}}\right)\right)$ as a function of excitation potential $(\chi)$ and wavelength $(\lambda)$. The initial values of $\log g$ were tuned to minimize the disagreement between the neutral and ionized species abundances of $\mathrm{Ti}, \mathrm{Cr}$, and $\mathrm{Fe}$ (particular attention was paid to the $\mathrm{Fe}$ data). Lastly, the microturbulent velocities $v_{\mathrm{t}}$ were set so as to reduce any dependence on abundance as a function of EW. Final values of $T_{\text {eff }}, \log g, v_{\mathrm{t}}$, and metallicity $[\mathrm{Fe} / \mathrm{H}]$ are listed in Table 1 , as well as those values previously derived by Sneden et al. (2000a) for the RGB stars and Preston et al. (2006) for the RHB stars.

\subsection{Selection of Model Type}

To conduct a standard abundance analysis under the fundamental assumptions of one-dimensionality and LTE, two grids of model atmospheres are generally employed: Kurucz-Castelli (Castelli \& Kurucz 2003; Kurucz 2005) and MARCS (Gustafsson et al. 2008). ${ }^{12}$ The model selection criteria were as follows: the reconciliation of the metallicity discrepancy between the RGB and RHB stars of M15, the derivation of (spectroscopic-based) atmospheric parameters in reasonable agreement with those found via photometry, and the attainment of ionization balance between the Fe I and $\mathrm{Fe}$ II transitions. For the RHB targets, interpolated models from the Kurucz-Castelli and MARCS grids were comparable and yielded extremely similar abundance results. However, there are a few notable differences between the two model types for the RGB stars with regard to the $P_{\text {gas }}$ and $P_{\text {electron }}$ content pressures. Though beyond the scope of the current effort, it would be of considerable interest to examine in detail the exact departures between the Kurucz-Castelli and MARCS grids. To best achieve the aforementioned goals for the M15 data set, MARCS models were accordingly chosen.

\footnotetext{
12 Kurucz models are available through the Web site:

http://kurucz.harvard.edu/ and MARCS models can be downloaded via the Web site: http://marcs.astro.uu.se/
} 
Table 1

Photometry and Model Atmosphere Parameters

\begin{tabular}{|c|c|c|c|c|c|c|c|c|c|c|c|c|}
\hline Star & $V^{\mathrm{c}}$ & $V-K^{\mathrm{d}}$ & $\begin{array}{c}\text { Current-PHOTO }^{\mathrm{a}} \\
T_{\text {eff }} \\
(\mathrm{K}) \\
\end{array}$ & $\log (g)$ & $\begin{array}{c}\text { Current-SPEC } \\
T_{\text {eff }} \\
(\mathrm{K})\end{array}$ & $\log (g)$ & {$[\mathrm{M} / \mathrm{H}]$} & $\begin{array}{c}v_{\text {turb }} \\
\left(\mathrm{km} \mathrm{s}^{-1}\right)\end{array}$ & $\begin{array}{c}\text { Previous-SPEC } \\
T_{\text {eff }} \\
(\mathrm{K}) \\
\end{array}$ & $\log (g)$ & {$[\mathrm{M} / \mathrm{H}]$} & $\begin{array}{c}v_{\text {turb }} \\
\left(\mathrm{km} \mathrm{s}^{-1}\right)\end{array}$ \\
\hline \multirow[t]{2}{*}{ K341 } & 12.81 & 3.115 & 4343 & 0.88 & 4375 & 0.30 & -2.25 & 2.00 & 4200 & 0.15 & -2.20 & 2.15 \\
\hline & & & & & & & & & 4225 & 0.30 & -2.20 & 1.85 \\
\hline \multirow[t]{2}{*}{ K462 } & 12.90 & 3.178 & 4298 & 0.89 & 4400 & 0.30 & -2.25 & 2.00 & 4225 & 0.15 & -2.20 & 2.10 \\
\hline & & & & & & & & & 4275 & 0.45 & -2.20 & 2.00 \\
\hline \multirow[t]{2}{*}{ K583 } & 12.83 & 3.261 & 4241 & 0.82 & 4375 & 0.30 & -2.25 & 1.90 & 4275 & 0.15 & -2.20 & 2.20 \\
\hline & & & & & & & & & 4275 & 0.30 & -2.20 & 1.90 \\
\hline B009 & 15.20 & 2.091 & 5443 & 2.26 & 5300 & 1.65 & -2.50 & 2.60 & 5300 & 1.65 & -2.30 & 2.50 \\
\hline B028 & 15.88 & 1.907 & 5718 & 2.65 & 5750 & 2.40 & -2.50 & 2.85 & 5750 & 2.40 & -2.50 & 2.75 \\
\hline B224 & 15.69 & 1.775 & 5932 & 2.65 & 5600 & 2.10 & -2.50 & 2.60 & 5600 & 2.10 & -2.50 & 2.60 \\
\hline B262 & 15.28 & 2.330 & 5122 & 2.16 & 4950 & 1.30 & -2.50 & 1.90 & 5000 & 1.50 & -2.30 & 1.70 \\
\hline B412 & 15.81 & 1.888 & 5748 & 2.63 & 6200 & 2.70 & -2.50 & 3.30 & 6250 & 2.70 & -2.50 & 3.30 \\
\hline B584 & 15.53 & 1.655 & 6142 & 2.66 & 6000 & 2.70 & -2.50 & 2.90 & 6000 & 2.70 & -2.30 & 2.70 \\
\hline
\end{tabular}

Notes.

a These values are derived with the IRFM effective temperature formulations (Equations (8) and (9)) of Alonso et al. (1999).

b The previous values for the spectroscopic parameters for the RGB stars are taken from Sneden et al. (2000a, 1997), respectively, and those for the RHB stars are obtained from Preston et al. (2006).

c The RGB $V$ magnitudes are taken from K. M. Cudworth (2011, private communication) and those for the RHB targets are obtained from Buonanno et al. (1983).

d The $K$ magnitudes for all members of the M15 data set are taken from the 2MASS database (Skrutskie et al. 2006).

Table 2

Element Abundances for Individual Transitions in M15 RGB Stars

\begin{tabular}{|c|c|c|c|c|c|c|c|c|c|}
\hline $\begin{array}{c}\text { Element/ } \\
\text { Ionization State }\end{array}$ & Species & $\begin{array}{c}\text { Measurement } \\
\text { Type }\end{array}$ & $\begin{array}{c}\text { Wavelength } \\
{[\AA]}\end{array}$ & $\begin{array}{c}\chi \\
{[\mathrm{eV}]}\end{array}$ & $\log (g f)$ & $\log \epsilon_{\mathrm{K} 341}$ & $\log \epsilon_{\mathrm{K} 462}$ & $\log \epsilon_{\mathrm{K} 583}$ & $\begin{array}{c}g f \\
\text { Reference }\end{array}$ \\
\hline $\mathrm{C} \mathrm{I}(\mathrm{CH})$ & 6.0 & SYNTH & $\approx 4300.000$ & $\ldots$ & $\ldots$ & 5.51 & 5.76 & 5.41 & Plez \& Cohen (2005) \\
\hline O I & 8.0 & SYNTH & 6300.304 & 0.000 & -9.776 & 7.03 & 6.88 & 6.75 & Baluja \& Zeippen (1988) \\
\hline $\mathrm{O} \mathrm{I}$ & 8.0 & SYNTH & 6363.776 & 0.020 & -10.257 & 7.01 & 7.01 & $\ldots$ & Baluja \& Zeippen (1988) \\
\hline $\mathrm{Na} \mathrm{I}$ & 11.0 & EW & 5889.950 & 0.000 & 0.108 & 3.45 & 3.61 & 4.70 & Volz et al. (1996) \\
\hline $\mathrm{Na} \mathrm{I}$ & 11.0 & EW & 5895.920 & 0.000 & -0.194 & $\ldots$ & 3.42 & 4.70 & Volz et al. (1996) \\
\hline $\mathrm{Mg} \mathrm{I}$ & 12.0 & EW & 4571.100 & 0.000 & -5.623 & 5.39 & 5.42 & 5.33 & Fischer \& Tachiev (2003) \\
\hline $\mathrm{Mg} \mathrm{I}$ & 12.0 & EW & 4702.990 & 4.346 & -0.440 & 5.65 & 5.61 & 5.43 & Chang \& Tang (1990) \\
\hline $\mathrm{Mg} \mathrm{I}$ & 12.0 & EW & 5528.400 & 4.346 & -0.498 & 5.72 & 5.35 & 5.48 & Chang \& Tang (1990) \\
\hline Mg I & 12.0 & EW & 5711.088 & 4.346 & -1.724 & $\ldots$ & 5.34 & 5.49 & Chang \& Tang (1990) \\
\hline Al I & 13.0 & EW & 3944.010 & 0.000 & -0.638 & 4.18 & 4.23 & 4.54 & Mendoza et al. (1995) \\
\hline Al I & 13.0 & EW & 3961.520 & 0.010 & -0.336 & 3.93 & 3.99 & 4.31 & Mendoza et al. (1995) \\
\hline Si I & 14.0 & EW & 3905.523 & 1.910 & -1.041 & 5.11 & $\ldots$ & 5.42 & O’Brian \& Lawler (1991) \\
\hline Si I & 14.0 & EW & 4102.936 & 1.910 & -3.336 & 5.51 & 5.76 & 5.61 & Fischer (2004) \\
\hline Si I & 14.0 & EW & 5684.484 & 4.954 & -1.420 & 5.16 & $\ldots$ & $\ldots$ & Nahar \& Pradhan (1993) \\
\hline Si I & 14.0 & EW & 5690.425 & 4.930 & -1.870 & 5.43 & $\ldots$ & 5.40 & Garz (1973) \\
\hline $\mathrm{Ca} \mathrm{I}$ & 20.0 & EW & 4425.440 & 1.879 & -0.358 & 3.91 & 4.02 & 3.87 & Fuhr \& Wiese (1998) \\
\hline $\mathrm{Ca} \mathrm{I}$ & 20.0 & EW & 5588.760 & 2.526 & 0.210 & 3.94 & 3.83 & 3.89 & Köstlin (1964) \\
\hline $\mathrm{Ca} \mathrm{I}$ & 20.0 & EW & 5857.460 & 2.933 & 0.230 & 4.07 & 3.99 & 4.06 & Köstlin (1964) \\
\hline $\mathrm{Ca} \mathrm{I}$ & 20.0 & EW & 6102.727 & 1.879 & -0.790 & $\ldots$ & 4.15 & 3.97 & Fuhr \& Wiese (1998) \\
\hline $\mathrm{Ca} \mathrm{I}$ & 20.0 & EW & 6122.230 & 1.886 & -0.315 & 4.19 & 4.00 & 4.03 & Fuhr \& Wiese (1998) \\
\hline $\mathrm{Ca} \mathrm{I}$ & 20.0 & EW & 6162.180 & 1.900 & -0.089 & 4.19 & 3.93 & 4.17 & Fuhr \& Wiese (1998) \\
\hline $\mathrm{Ca} \mathrm{I}$ & 20.0 & EW & 6439.070 & 2.526 & 0.470 & $\ldots$ & 4.02 & 4.15 & Köstlin (1964) \\
\hline $\mathrm{Ca} \mathrm{I}$ & 20.0 & EW & 6717.690 & 2.709 & -0.524 & 4.29 & 4.14 & 4.13 & Fuhr \& Wiese (1998) \\
\hline
\end{tabular}

(This table is available in its entirety in machine-readable and Virtual Observatory (VO) forms in the online journal. A portion is shown here for guidance regarding its form and content.)

\subsection{Persistent Metallicity Disagreement between $R G B$ and RHB Stars}

For the RHB stars, the presently derived metallicities differ slightly from those of Preston et al. (2006): $\left\langle\left[\mathrm{Fe}_{\mathrm{I}} / \mathrm{H}\right]\right\rangle=-2.69$ (a change of $\Delta=-0.03$ ) and $\left\langle\left[\mathrm{Fe}_{\mathrm{II}} / \mathrm{H}\right]\right\rangle=-2.64$ (a change of $\Delta=-0.04)$. However, for the RGB stars, the $[\mathrm{Fe} / \mathrm{H}]$ results of the current study do vary significantly from those of Sneden et al. (2000a): $\left\langle\left[\mathrm{Fe}_{\mathrm{I}} / \mathrm{H}\right]\right\rangle=-2.56$ (a downward revision of $\Delta=-0.26$ ) and $\left\langle\left[\mathrm{Fe}_{\mathrm{II}} / \mathrm{H}\right]\right\rangle=-2.53$ (a downward revision of $\Delta=-0.28$ ). The remaining metallicity discrepancy between the RGB and RHB stars is as follows: $\Delta(\mathrm{RGB}-\mathrm{RHB})_{\mathrm{Fe}}=0.13$ and $\Delta(\mathrm{RGB}-\mathrm{RHB})_{\mathrm{Fe} \text { II }}=0.11$. Even with the employment of MARCS models and the incorporation of Rayleigh scattering (not done in previous efforts), the offset persists. Repeated exercises with variations in the $T_{\text {eff }}, \log g$, and $v_{\mathrm{t}}$ values showed that this metallicity disagreement in all likelihood cannot be attributed to differences in these atmospheric parameters. As a further check, the derivation of $\left[\mathrm{Fe}_{\mathrm{I}, \mathrm{II}} / \mathrm{H}\right]$ values was performed with a list of transitions satisfactorily measurable in both RGB and RHB spectra. No reduction in the metallicity disagreement was seen as the offsets were found to be: $\Delta(\mathrm{RGB}-\mathrm{RHB})_{\mathrm{Fe}}=$ 
Table 3

Element Abundances for Individual Transitions in M15 RHB Stars

\begin{tabular}{|c|c|c|c|c|c|c|c|c|c|c|c|c|}
\hline $\begin{array}{c}\text { Element/ } \\
\text { Ionization State }\end{array}$ & Species & $\begin{array}{c}\text { Measurement } \\
\text { Type }\end{array}$ & $\begin{array}{c}\lambda \\
{[\AA ̊ \cap]}\end{array}$ & $\begin{array}{c}\chi \\
{[\mathrm{eV}]}\end{array}$ & $\log (g f)$ & $\log \epsilon_{\mathrm{B} 009}$ & $\log \epsilon_{\mathrm{B} 028}$ & $\log \epsilon_{\mathrm{B} 224}$ & $\log \epsilon_{\mathrm{B} 262}$ & $\log \epsilon_{\mathrm{B} 412}$ & $\log \epsilon_{\mathrm{B} 584}$ & $\begin{array}{c}g f \\
\text { Reference }\end{array}$ \\
\hline $\mathrm{Na} \mathrm{I}$ & 11.0 & EW & 5889.950 & 0.000 & 0.112 & 5.09 & 4.05 & 4.67 & 4.63 & 3.81 & 3.86 & Volz et al. (1996) \\
\hline $\mathrm{Na} \mathrm{I}$ & 11.0 & EW & 5895.920 & 0.000 & -0.191 & 5.07 & 3.88 & 4.54 & 4.58 & 3.79 & 3.75 & Volz et al. (1996) \\
\hline $\mathrm{Mg} \mathrm{I}$ & 12.0 & EW & 3829.360 & 2.710 & -0.227 & 4.78 & 5.37 & 5.35 & $\ldots$ & $\ldots$ & 5.38 & Fischer \& Tachiev (2003) \\
\hline $\mathrm{Mg} \mathrm{I}$ & 12.0 & EW & 3832.310 & 2.710 & -0.353 & 4.75 & 5.42 & 5.37 & 5.04 & $\ldots$ & 5.16 & Fischer \& Tachiev (2003) \\
\hline Mg I & 12.0 & EW & 4571.100 & 0.000 & -5.623 & 4.90 & 5.31 & 5.26 & 5.19 & 5.34 & 5.57 & Fischer \& Tachiev (2003) \\
\hline $\mathrm{Mg} \mathrm{I}$ & 12.0 & EW & 5172.684 & 2.712 & -0.393 & 4.73 & 5.61 & 5.44 & 5.34 & 5.57 & 5.52 & Fischer \& Tachiev (2003) \\
\hline $\mathrm{Mg} \mathrm{I}$ & 12.0 & EW & 5183.604 & 2.717 & -0.167 & 4.91 & 5.62 & $\ldots$ & 5.52 & 5.76 & 5.57 & Fischer \& Tachiev (2003) \\
\hline $\mathrm{Mg} \mathrm{I}$ & 12.0 & EW & 5528.420 & 4.346 & -0.498 & 4.92 & 5.25 & 5.33 & 5.14 & 5.26 & 5.37 & Chang \& Tang (1990) \\
\hline Al I & 13.0 & EW & 3944.010 & 0.000 & -0.638 & 4.09 & 3.21 & 3.42 & 3.50 & 3.31 & 3.22 & Mendoza et al. (1995) \\
\hline Al I & 13.0 & EW & 3961.530 & 0.014 & -0.336 & 3.94 & 3.01 & 3.30 & 3.19 & 3.16 & 3.13 & Mendoza et al. (1995) \\
\hline Si I & 14.0 & EW & 3905.523 & 1.910 & -1.041 & $\ldots$ & 5.11 & 5.30 & 5.29 & 4.98 & 4.87 & O’Brian \& Lawler (1991) \\
\hline Si I & 14.0 & EW & 4102.936 & 1.910 & -3.336 & 5.24 & $\ldots$ & $\ldots$ & $\ldots$ & $\ldots$ & $\ldots$ & Fischer (2004) \\
\hline $\mathrm{Ca} \mathrm{I}$ & 20.0 & EW & 4226.740 & 0.000 & 0.244 & 4.05 & 4.29 & 4.36 & 3.70 & 4.29 & 4.22 & Fuhr \& Wiese (1998) \\
\hline $\mathrm{Ca} \mathrm{I}$ & 20.0 & EW & 5588.760 & 2.526 & 0.210 & 4.21 & 4.02 & 4.12 & 3.97 & 4.18 & $\ldots$ & Köstlin (1964) \\
\hline $\mathrm{Ca} \mathrm{I}$ & 20.0 & EW & 5857.460 & 2.933 & 0.230 & 4.25 & $\ldots$ & 4.14 & 4.24 & $\ldots$ & $\ldots$ & Köstlin (1964) \\
\hline $\mathrm{Ca} \mathrm{I}$ & 20.0 & EW & 6102.727 & 1.879 & -0.790 & 4.19 & $\ldots$ & $\ldots$ & 3.97 & 4.27 & $\ldots$ & Fuhr \& Wiese (1998) \\
\hline $\mathrm{Ca} \mathrm{I}$ & 20.0 & EW & 6122.230 & 1.886 & -0.315 & 4.22 & 4.22 & 4.14 & 4.12 & 4.15 & $\ldots$ & Fuhr \& Wiese (1998) \\
\hline $\mathrm{Ca} \mathrm{I}$ & 20.0 & EW & 6162.180 & 1.900 & -0.089 & 4.29 & 4.20 & 4.08 & 4.20 & 4.20 & 4.13 & Fuhr \& Wiese (1998) \\
\hline $\mathrm{Ca} \mathrm{I}$ & 20.0 & EW & 6439.070 & 2.526 & 0.470 & 4.01 & 4.26 & 4.01 & 4.05 & 4.08 & $\ldots$ & Köstlin (1964) \\
\hline $\mathrm{Ca} \mathrm{I}$ & 20.0 & EW & 6493.780 & 2.521 & 0.140 & 3.91 & $\ldots$ & 3.97 & $\ldots$ & $\ldots$ & $\ldots$ & Köstlin (1964) \\
\hline $\mathrm{Ca} \mathrm{I}$ & 20.0 & EW & 6499.650 & 2.523 & -0.590 & 4.19 & $\ldots$ & . & $\ldots$ & $\ldots$ & $\ldots$ & Köstlin (1964) \\
\hline $\mathrm{Ca} \mathrm{I}$ & 20.0 & EW & 6717.690 & 2.709 & -0.524 & $\ldots$ & $\ldots$ & $\ldots$ & 4.24 & $\ldots$ & $\ldots$ & Fuhr \& Wiese (1998) \\
\hline
\end{tabular}

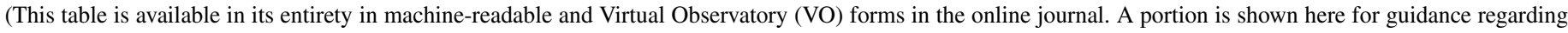
its form and content.)

0.11 (with 45 candidate $\mathrm{Fe}_{\mathrm{I}}$ lines) and $\Delta(\mathrm{RGB}-\mathrm{RHB})_{\mathrm{Fe} \text { II }}=$ 0.14 (with 3 candidate Fe $\mathrm{IIII}_{\mathrm{II}}$ lines).

The data from the M15 RGB and RHB stars originate from different telescope/instrument setups. Additionally, somewhat different data reduction procedures were employed for the two samples. Possible contributors to the iron abundance offset could be the lack of consideration of spherical symmetry in the line transfer computations and the generation of sufficiently representative stellar atmospheric models for these highly evolved stars (which exist at the very tip of the giant branch and/or have undergone He-core flash). Indeed, it is difficult to posit a single, clear-cut explanation for the disparity in the RGB and RHB $[\mathrm{Fe} / \mathrm{H}]$ values. In an analysis of the GC M92, King et al. (1998) derived an average abundance ratio of $\langle[\mathrm{Fe} / \mathrm{H}]\rangle=-2.52$ for six subgiant stars, a factor of two lower than the $\langle[\mathrm{Fe} / \mathrm{H}]\rangle$ value measured in the red giant stars. Similarly, Korn et al. (2007) surveyed turn off (TO) and RGB stars of NGC 6397 and found a metallicity offset of about 0.15 dex (with the TO stars reporting consistently lower values of $[\mathrm{Fe} / \mathrm{H}])$. They argued that the TO stars were afflicted by gravitational settling and other mixing processes and as a result, the $\mathrm{Fe}$ abundances of giant stars were likely to be nearer to the true value. While the TO stars do have $T_{\text {eff }}$ values close to that of the M15 RHB stars, they have surface gravities and lifetimes that are considerably larger. Accordingly, it is not clear if the offset in M15 has a physical explanation similar to that proposed in the case of NGC 6397.

\section{ABUNDANCE RESULTS}

For the extraction of abundances, the two filters of line strength and contaminant presence were used to assemble an effective line list. Abundance derivations for the majority of elements employed the technique of synthetic spectrum line profile fitting (accomplished with the updated MOOG code as described in Section 3.2). For a small group of elements (those whose associated spectral features lack both hyperfine and isotopic structure), the simplified approach of EW measurement was used (completed with both the MOOG code and the SPECTRE program; Fitzpatrick \& Sneden 1987). Presented in Tables 2 and 3 are the $\log \epsilon$ abundance values for the individual transitions detected in the M15 RGB and RHB stars, respectively. These tables also list the relevant line parameters as well as the associated literature references for the $g f$-values employed.

In addition to the line-to-line scatter, errors in the abundance results may arise due to uncertainties in the model atmospheric parameters. To quantify these errors in the M15 data set, an RGB target, K462, is first selected. If alterations of $\Delta T_{\text {eff }}= \pm 100 \mathrm{~K}$ are applied, then the abundances of neutral species change by approximately $\Delta\left[\mathrm{X}_{\mathrm{I}} / \mathrm{H}\right] \simeq \pm 0.15$, whereas the abundances of singly ionized species change by about $\Delta\left[\mathrm{X}_{\mathrm{II}} / \mathrm{H}\right] \simeq \pm 0.04$. Variations of $\Delta \log g= \pm 0.20$ yield $\Delta\left[\mathrm{X}_{\mathrm{I}} / \mathrm{H}\right] \simeq \pm 0.04$ in neutral species abundances and $\Delta\left[\mathrm{X}_{\mathrm{II}} / \mathrm{H}\right] \simeq \pm 0.05$ in singly ionized species abundances. Changes in the microturbulent velocity on the order of $\Delta v_{\mathrm{t}}= \pm 0.20$ result in abundance variations of $\Delta\left[\mathrm{X}_{\mathrm{I}} / \mathrm{H}\right] \simeq \pm 0.08$ and $\Delta\left[\mathrm{X}_{\mathrm{II}} / \mathrm{H}\right] \simeq \pm 0.04$. The exact same procedure is then repeated for the RHB star, B009. Modifications of the temperature by $\Delta T_{\text {eff }}= \pm 100 \mathrm{~K}$ lead to abundance changes of $\Delta\left[\mathrm{X}_{\mathrm{I}} / \mathrm{H}\right] \simeq \pm 0.09$ in neutral species and $\Delta\left[\mathrm{X}_{\text {II }} / \mathrm{H}\right] \simeq \pm 0.02$ in singly ionized species. Alterations of the surface gravity by $\Delta \log g= \pm 0.20$ engender variations of $\Delta\left[\mathrm{X}_{\mathrm{I}} / \mathrm{H}\right] \simeq \pm 0.01$ and $\Delta\left[\mathrm{X}_{\mathrm{II}} / \mathrm{H}\right] \simeq \pm 0.07$. Finally, variations of $\Delta v_{\mathrm{t}}= \pm 0.20$ produce abundance changes of $\Delta\left[\mathrm{X}_{\mathrm{I}} / \mathrm{H}\right] \simeq \pm 0.08$ and $\Delta\left[\mathrm{X}_{\mathrm{II}} / \mathrm{H}\right] \simeq \pm 0.04$.

To discuss the abundance results in the following subsections, the elements are divided into four groupings: light $(Z=8 ; 11 \leqslant Z \leqslant 21$; Figure 3$)$, iron-peak $(22 \leqslant Z \leqslant$ 28; Figure 4), light/intermediate $n$-capture $(29 \leqslant Z \leqslant 59$; Figure 5), and heavy/other ( $60 \leqslant Z \leqslant 70 ; Z=72,76,77,90$; Figure 6). The measurement of abundances was completed for 


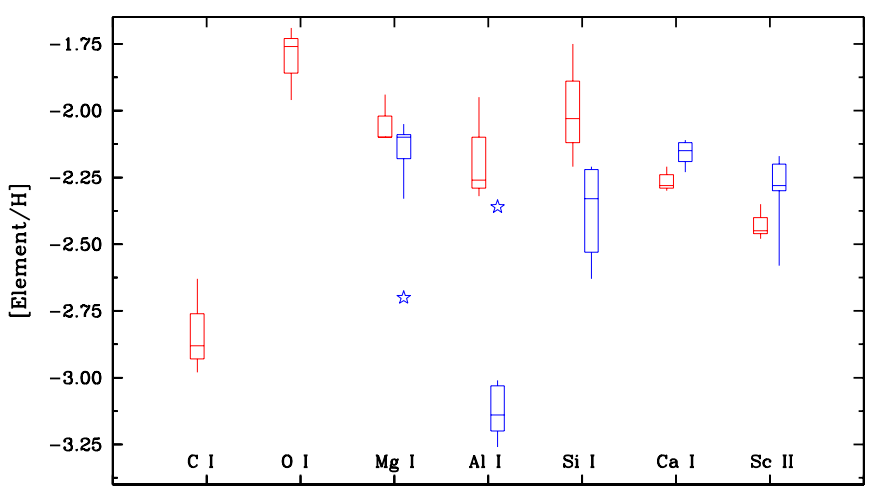

Element

Figure 3. Binned abundances for the elements with $Z=6,8,12-14,20-21$ displayed as quartile box plots. For each species, the box plot shows the median, the upper and lower quartiles, and the extremes for both the RGB (denoted in red) and RHB (signified in blue) samples. Outliers, data points with values greater than 1.25 times the median, are indicated by star symbols. Note that there are no RHB points for $\mathrm{CI}_{\mathrm{I}}$ and $\mathrm{O}$ I. Note that a depletion in the relative carbon abundance with respect to solar is found.

(A color version of this figure is available in the online journal.)

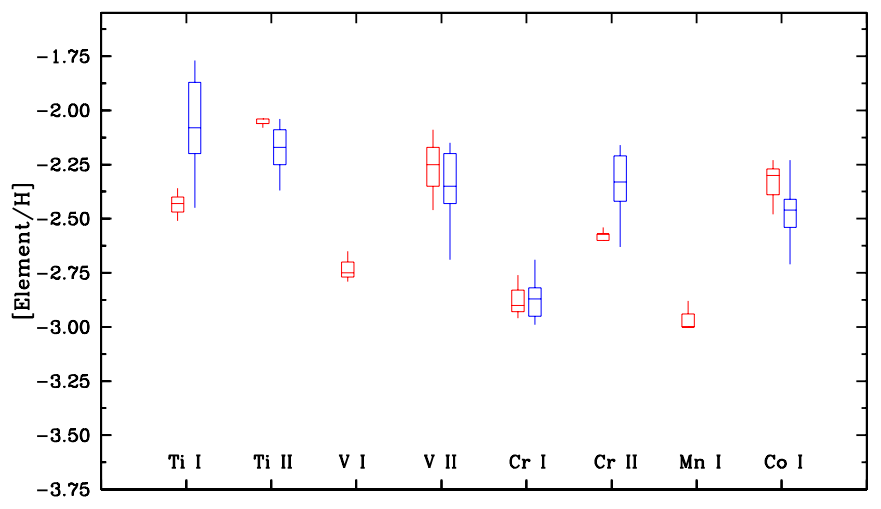

Element

Figure 4. Binned abundances for the elements with $22 \leqslant Z \leqslant 27$ displayed as quartile box plots. For each species, the box plot shows the median, the upper and lower quartiles, and the extremes for both the RGB (denoted in red) and RHB (signified in blue) samples. Note that no RHB data exist for V I. Also, the Mn I results have been set aside for the RHB stars (consult the text for further information). The most consistent agreement between neutral and first-ionized species is found for Ti in all M15 stars.

(A color version of this figure is available in the online journal.)

a total of 40 species. Note that for the elements $\mathrm{Sc}, \mathrm{Ti}, \mathrm{V}$, and $\mathrm{Cr}$, abundance determinations were possible for both the neutral and first-ionized species. In light of Saha-Boltzmann calculations for these elements, greater weight is given to the singly ionized abundances (i.e., for the stars of the M15 data set, only a small fraction of these elements predominantly reside in the neutral state).

Figures 3-6 exhibit the abundance ratios for the M15 sample in the form of quartile box plots. These plots show the interquartile range, the median, and the minimum/maximum of the data. Outliers, points which have a value greater than 1.25 times the median, are also indicated. For all of the figures, RGB abundances are signified in red while RHB abundances are denoted in blue. Note that the plots depict the abundance results in the $[$ Elem $/ \mathrm{H}]$ form in order to preclude erroneous comparisons of the RGB and RHB data, which would arise from the iron abundance offset between the two groups.

Table 4 contains the $\langle[$ Elem/Fe $]\rangle$ values for elements analyzed in the M15 sample along with the line-to-line scatter

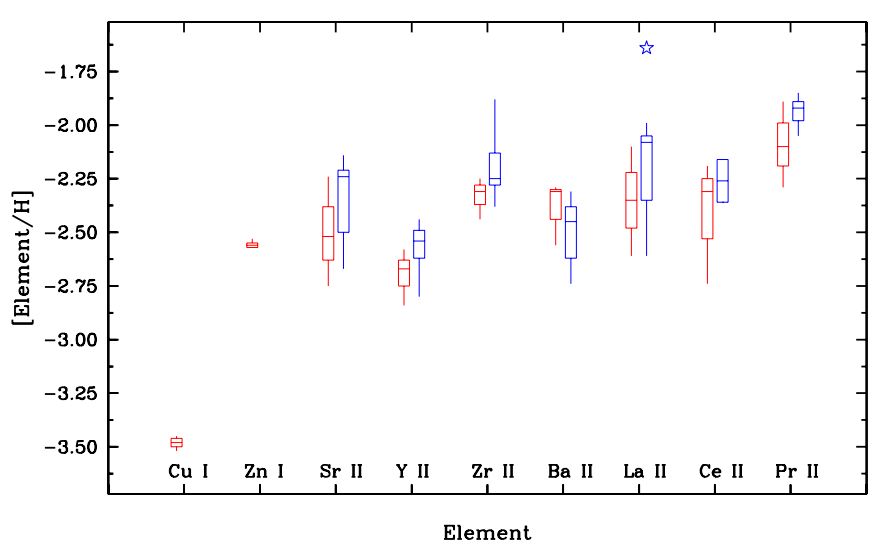

Figure 5. Binned abundances for the elements with $Z=29,30,38-40,56-59$ displayed as quartile box plots. For each species, the box plot shows the median, the upper and lower quartiles, and the extremes for both the RGB (denoted in red) and RHB (signified in blue) samples. Outliers, data points with values greater than 1.25 times the median, are indicated by star symbols. Note that there are no RHB data points for $\mathrm{Cu}$ I and also, only one RHB abundance value for $\mathrm{ZnI}$.

(A color version of this figure is available in the online journal.)

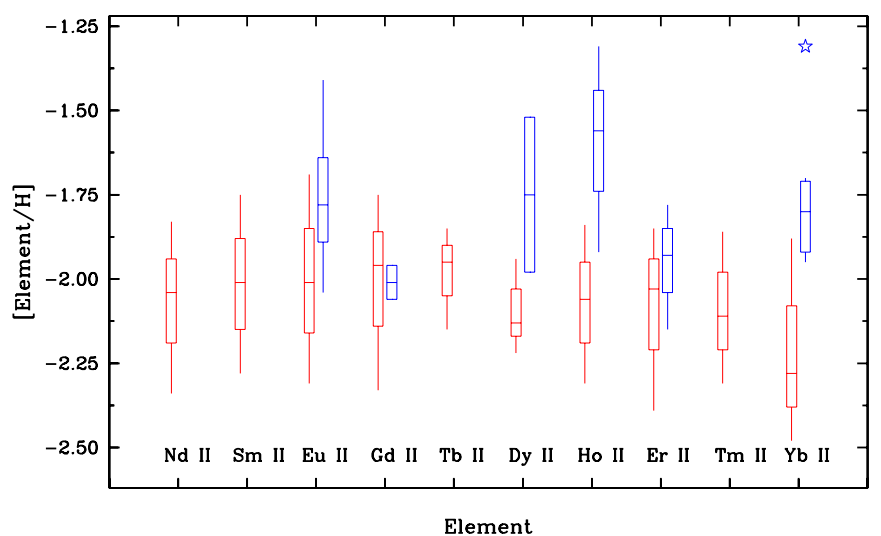

Figure 6. Binned abundances for the elements with $60 \leqslant Z \leqslant 70$ displayed as quartile box plots. For each species, the box plot shows the median, the upper and lower quartiles, and the extremes for both the RGB (denoted in red) and RHB (signified in blue) samples. Outliers, data points with values greater than 1.25 times the median, are indicated by star symbols. Note that only one RHB abundance value was found for the elements Nd II, Sm II, and Tm II. In the case of Tb II, no RHB measurements were possible.

(A color version of this figure is available in the online journal.)

(given in the form of standard deviations), and the number of lines employed. The subsequent discussion will generally refer to these table data and as is customary, present the relative element abundances with associated $\sigma$ values. The reference solar photospheric abundances (without non-LTE correction) are largely taken from the three-dimensional analyses of Asplund et al. (2005, 2009) and Grevesse et al. (2010). However, the photospheric values for some of the $n$-capture elements are obtained from other investigations (e.g., Lawler et al. 2009). Table 5 lists all of the chosen $\log \epsilon_{\odot}$ numbers. Note that in the derivation of the relative element abundance ratios $[\mathrm{X} / \mathrm{Fe}],\left\langle\left[\mathrm{Fe}_{\mathrm{I}} / \mathrm{H}\right]\right\rangle$ are employed for the neutral species transitions while $\left\langle\left[\mathrm{Fe}_{\mathrm{II}} / \mathrm{H}\right]\right\rangle$ are used for the singly ionized lines. This is done in order to minimize ionization equilibrium uncertainties as described in detail by Kraft \& Ivans (2003).

\subsection{General Abundance Trends}

Within the RGB sample, the neutron-capture element abundances of K462 are consistently the largest whereas those of K583 are the smallest. The two RHB stars, B009 and B262, 
Table 4

Average Relative Abundance Data for M15 Program Stars

\begin{tabular}{|c|c|c|c|c|c|c|c|c|c|}
\hline$\langle[$ Elem $/$ Fe I,II $]\rangle$ & K341 & K462 & K583 & B009 & B028 & B224 & B262 & B412 & B584 \\
\hline$\langle[\mathrm{C} \mathrm{I} / \mathrm{Fe} \mathrm{I}]\rangle$ & -0.34 & -0.08 & -0.40 & -0.10 & $\ldots$ & $\ldots$ & $\ldots$ & $\ldots$ & $\ldots$ \\
\hline$\sigma(\log \epsilon(\mathrm{C} \mathrm{I}))$ & $\ldots$ & $\ldots$ & $\ldots$ & $\ldots$ & $\ldots$ & $\ldots$ & $\ldots$ & $\ldots$ & $\ldots$ \\
\hline No. of Lines & 1 & 1 & 1 & 1 & 0 & 0 & 0 & 0 & 0 \\
\hline$\langle[\mathrm{O} \mathrm{I} / \mathrm{Fe} \mathrm{I}]\rangle$ & 0.85 & 0.78 & 0.62 & $\ldots$ & $\ldots$ & $\ldots$ & $\ldots$ & $\ldots$ & $\ldots$ \\
\hline$\sigma(\log \epsilon(\mathrm{OI}))$ & 0.01 & 0.09 & $\ldots$ & $\ldots$ & $\ldots$ & $\ldots$ & $\ldots$ & $\ldots$ & $\ldots$ \\
\hline No. of Lines & 2 & 2 & 1 & 0 & 0 & 0 & 0 & 0 & 0 \\
\hline$\langle[\mathrm{NaI} / \mathrm{Fe} \mathrm{I}]\rangle$ & -0.18 & -0.11 & 1.11 & 1.60 & 0.46 & 1.18 & 1.21 & 0.25 & 0.28 \\
\hline$\sigma(\log \epsilon(\mathrm{Na} \mathrm{I}))$ & $\ldots$ & 0.13 & 0.00 & 0.01 & 0.12 & 0.09 & 0.04 & 0.01 & 0.08 \\
\hline No. of Lines & 1 & 2 & 2 & 2 & 2 & 2 & 2 & 2 & 2 \\
\hline$\left\langle\left[\mathrm{Mg}_{\mathrm{I}} / \mathrm{Fe}_{\mathrm{I}}\right]\right\rangle$ & 0.60 & 0.45 & 0.48 & -0.01 & 0.56 & 0.56 & 0.44 & 0.57 & 0.54 \\
\hline$\sigma\left(\log \epsilon\left(\mathrm{Mg}_{\mathrm{I}}\right)\right)$ & 0.17 & 0.13 & 0.07 & 0.09 & 0.15 & 0.07 & 0.20 & 0.23 & 0.16 \\
\hline No. of Lines & 3 & 4 & 4 & 6 & 2 & 1 & 2 & 2 & 2 \\
\hline$\langle[\mathrm{Al} \mathrm{I} / \mathrm{Fe} \mathrm{I}]\rangle$ & 0.23 & 0.29 & 0.64 & 0.34 & -0.60 & -0.27 & -0.26 & -0.52 & -0.52 \\
\hline$\sigma(\log \epsilon(\mathrm{Al} \mathrm{I}))$ & 0.18 & 0.17 & 0.16 & 0.11 & 0.14 & 0.08 & 0.22 & 0.11 & 0.06 \\
\hline No. of Lines & 2 & 2 & 2 & 2 & 2 & 2 & 2 & 2 & 2 \\
\hline$\langle[\mathrm{Si} \mathrm{I} / \mathrm{Fe} \mathrm{I}]\rangle$ & 0.33 & 0.80 & 0.55 & 0.42 & 0.26 & 0.53 & 0.55 & 0.09 & 0.00 \\
\hline$\sigma(\log \epsilon(\operatorname{Si} \mathrm{I}))$ & 0.20 & 0.08 & 0.01 & $\ldots$ & $\ldots$ & $\ldots$ & $\ldots$ & $\ldots$ & $\ldots$ \\
\hline No. of Lines & 4 & 2 & 2 & 1 & 1 & 1 & 1 & 1 & 1 \\
\hline$\langle[\mathrm{Ca} \mathrm{I} / \mathrm{Fe} \mathrm{I}]\rangle$ & 0.33 & 0.25 & 0.30 & 0.53 & 0.55 & 0.55 & 0.54 & 0.51 & 0.51 \\
\hline$\sigma(\log \epsilon(\mathrm{Ca} \mathrm{I}))$ & 0.15 & 0.10 & 0.12 & 0.13 & 0.11 & 0.13 & 0.18 & 0.08 & 0.06 \\
\hline No. of Lines & 6 & 8 & 8 & 9 & 5 & 7 & 9 & 6 & 2 \\
\hline$\langle[\mathrm{Sc} \mathrm{I} / \mathrm{Fe} \mathrm{I}]\rangle$ & $\ldots$ & $\ldots$ & -0.76 & $\ldots$ & $\ldots$ & $\ldots$ & $\ldots$ & $\ldots$ & $\ldots$ \\
\hline$\sigma(\log \epsilon(\mathrm{Sc} \mathrm{I}))$ & $\ldots$ & $\ldots$ & $\ldots$ & $\ldots$ & $\ldots$ & $\ldots$ & $\ldots$ & $\ldots$ & $\ldots$ \\
\hline No. of Lines & 0 & 0 & 1 & 0 & 0 & 0 & 0 & 0 & 0 \\
\hline$\langle[\mathrm{Sc}$ II $/ \mathrm{Fe}$ II $]\rangle$ & 0.16 & 0.04 & 0.12 & 0.37 & 0.43 & 0.37 & 0.16 & 0.39 & 0.32 \\
\hline$\sigma(\log \epsilon(\mathrm{Sc}$ II $))$ & 0.10 & 0.12 & 0.10 & 0.16 & 0.06 & 0.08 & 0.09 & 0.20 & $\ldots$ \\
\hline No. of Lines & 2 & 2 & 2 & 2 & 3 & 3 & 4 & 2 & 1 \\
\hline$\langle[\mathrm{Ti} \mathrm{I} / \mathrm{Fe} \mathrm{I}]\rangle$ & 0.11 & 0.19 & 0.07 & 0.58 & 0.60 & 0.54 & 0.32 & 0.85 & 0.77 \\
\hline$\sigma(\log \epsilon(\mathrm{Ti} \mathrm{I}))$ & 0.12 & 0.13 & 0.11 & 0.16 & 0.16 & 0.15 & 0.15 & 0.08 & 0.14 \\
\hline No. of Lines & 15 & 12 & 13 & 2 & 4 & 4 & 6 & 2 & 2 \\
\hline$\langle[\mathrm{Ti}$ II $/ \mathrm{Fe}$ II $]\rangle$ & 0.47 & 0.44 & 0.47 & 0.38 & 0.48 & 0.40 & 0.31 & 0.52 & 0.50 \\
\hline$\sigma(\log \epsilon(\mathrm{Ti}$ II $))$ & 0.11 & 0.13 & 0.14 & 0.10 & 0.10 & 0.10 & 0.07 & 0.07 & 0.06 \\
\hline No. of Lines & 14 & 8 & 16 & 9 & 7 & 9 & 11 & 8 & 7 \\
\hline$\langle[\mathrm{V} \mathrm{I} / \mathrm{Fe} \mathrm{I}]\rangle$ & -0.26 & -0.11 & -0.18 & $\ldots$ & $\ldots$ & $\ldots$ & $\ldots$ & $\ldots$ & $\ldots$ \\
\hline$\sigma\left(\log \epsilon\left(\mathrm{V}_{\mathrm{I}}\right)\right)$ & 0.07 & 0.19 & 0.12 & $\ldots$ & $\ldots$ & $\ldots$ & $\ldots$ & $\ldots$ & $\ldots$ \\
\hline No. of Lines & 2 & 3 & 3 & 0 & 0 & 0 & 0 & 0 & 0 \\
\hline$\langle[\mathrm{V}$ II $/ \mathrm{Fe}$ II $]\rangle$ & 0.27 & 0.43 & 0.11 & 0.23 & 0.41 & 0.31 & 0.04 & $\ldots$ & 0.44 \\
\hline$\sigma\left(\log \epsilon\left(V_{\text {II }}\right)\right)$ & 0.01 & 0.21 & 0.21 & $\ldots$ & 0.05 & $\ldots$ & 0.04 & $\ldots$ & 0.03 \\
\hline No. of Lines & 2 & 2 & 2 & 1 & 2 & 1 & 2 & 0 & 2 \\
\hline$\langle[\mathrm{Cr} \mathrm{I} / \mathrm{Fe} \mathrm{I}]\rangle$ & -0.36 & -0.22 & -0.38 & -0.21 & -0.24 & -0.21 & -0.22 & -0.07 & -0.19 \\
\hline$\sigma(\log \epsilon(\mathrm{Cr} \mathrm{I}))$ & 0.05 & 0.12 & 0.07 & 0.15 & 0.05 & 0.12 & 0.21 & 0.10 & 0.09 \\
\hline No. of Lines & 5 & 6 & 5 & 4 & 3 & 3 & 8 & 3 & 2 \\
\hline$\langle[\mathrm{Cr}$ II $/ \mathrm{Fe}$ II $]\rangle$ & -0.06 & -0.02 & -0.03 & 0.33 & 0.37 & 0.24 & 0.10 & 0.43 & 0.38 \\
\hline$\sigma(\log \epsilon(\mathrm{Cr}$ II $))$ & 0.14 & 0.18 & 0.06 & 0.01 & 0.21 & $\ldots$ & 0.10 & 0.06 & 0.07 \\
\hline No. of Lines & 4 & 3 & 3 & 2 & 3 & 1 & 3 & 2 & 2 \\
\hline$\langle[\mathrm{Mn} \mathrm{I} / \mathrm{Fe} \mathrm{I}]\rangle$ & -0.34 & -0.45 & -0.42 & -0.70 & -0.74 & -0.80 & -0.78 & $\ldots$ & $\ldots$ \\
\hline$\sigma(\log \epsilon(\mathrm{Mn} \mathrm{I}))$ & 0.02 & 0.04 & $\ldots$ & $\ldots$ & $\ldots$ & $\ldots$ & $\ldots$ & $\ldots$ & $\ldots$ \\
\hline No. of Lines & 2 & 2 & 1 & 1 & 1 & 1 & 1 & 0 & 0 \\
\hline$\langle[\mathrm{Fe} \mathrm{I} / \mathrm{H}]\rangle$ & -2.53 & -2.54 & -2.57 & -2.69 & -2.65 & -2.74 & -2.77 & -2.62 & -2.63 \\
\hline$\sigma\left(\log \epsilon\left(\mathrm{Fe}_{\mathrm{I}}\right)\right)$ & 0.14 & 0.12 & 0.14 & 0.12 & 0.12 & 0.11 & 0.14 & 0.12 & 0.15 \\
\hline No. of Lines & 59 & 57 & 63 & 98 & 72 & 72 & 100 & 45 & 60 \\
\hline$\langle[\mathrm{Fe}$ II $/ \mathrm{H}]\rangle$ & -2.51 & -2.51 & -2.57 & -2.65 & -2.60 & -2.66 & -2.73 & -2.59 & -2.59 \\
\hline$\sigma(\log \epsilon(\mathrm{Fe}$ II $))$ & 0.13 & 0.11 & 0.10 & 0.09 & 0.07 & 0.13 & 0.13 & 0.07 & 0.13 \\
\hline No. of Lines & 8 & 9 & 10 & 12 & 8 & 9 & 11 & 6 & 9 \\
\hline$\langle[\mathrm{Co} \mathrm{I} / \mathrm{Fe} \mathrm{I}]\rangle$ & 0.07 & 0.25 & 0.35 & 0.45 & 0.18 & 0.18 & 0.04 & $\ldots$ & 0.22 \\
\hline$\sigma(\log \epsilon($ Co I $))$ & 0.21 & 0.18 & 0.20 & 0.32 & $\ldots$ & $\ldots$ & $\ldots$ & $\ldots$ & $\ldots$ \\
\hline No. of Lines & 2 & 2 & 2 & 2 & 1 & 1 & 1 & 0 & 1 \\
\hline$\langle[\mathrm{Ni}$ I $/ \mathrm{Fe}$ I $]\rangle$ & $\ldots$ & $\ldots$ & $\ldots$ & -0.23 & $\ldots$ & $\ldots$ & $\ldots$ & $\ldots$ & $\ldots$ \\
\hline$\sigma(\log \epsilon(\mathrm{Ni} \mathrm{I}))$ & $\ldots$ & $\ldots$ & $\ldots$ & 0.11 & $\ldots$ & $\ldots$ & $\ldots$ & $\ldots$ & $\ldots$ \\
\hline No. of Lines & 0 & 0 & 0 & 2 & 0 & 0 & 0 & 0 & 0 \\
\hline$\langle[\mathrm{Cu}$ I/Fe I $]\rangle$ & -0.92 & -0.88 & -0.92 & $\ldots$ & $\ldots$ & $\ldots$ & $\ldots$ & $\ldots$ & $\ldots$ \\
\hline$\sigma(\log \epsilon(\mathrm{Cu} \mathrm{I}))$ & $\ldots$ & $\ldots$ & $\ldots$ & $\ldots$ & $\ldots$ & $\ldots$ & $\ldots$ & $\ldots$ & $\ldots$ \\
\hline No. of Lines & 1 & 1 & 1 & 0 & 0 & 0 & 0 & 0 & 0 \\
\hline$\left\langle\left[\mathrm{Zn} I / \mathrm{Fe}_{\mathrm{I}}\right]\right\rangle$ & 0.02 & -0.01 & 0.01 & $\ldots$ & $\ldots$ & 0.49 & 0.27 & $\ldots$ & $\ldots$ \\
\hline$\sigma(\log \epsilon(\mathrm{ZnI}))$ & 0.07 & 0.07 & $\ldots$ & $\cdots$ & $\cdots$ & $\ldots$ & 0.06 & $\cdots$ & $\cdots$ \\
\hline No. of Lines & 2 & 2 & 2 & 0 & 0 & 1 & 2 & 0 & 0 \\
\hline
\end{tabular}


Table 4

(Continued)

\begin{tabular}{|c|c|c|c|c|c|c|c|c|c|}
\hline$\langle[$ Elem $/$ Fe I,II $]\rangle$ & K341 & K462 & K583 & B009 & B028 & B224 & B262 & B412 & B584 \\
\hline$\langle[\mathrm{Sr}$ II $/ \mathrm{Fe}$ II $]\rangle$ & -0.01 & 0.28 & -0.18 & 0.16 & 0.35 & 0.43 & 0.06 & 0.38 & 0.46 \\
\hline$\sigma(\log \epsilon(\operatorname{Sr}$ II $))$ & 0.00 & 0.04 & 0.11 & 0.04 & 0.11 & 0.14 & 0.11 & 0.14 & 0.11 \\
\hline No. of Lines & 2 & 2 & 2 & 2 & 2 & 2 & 2 & 2 & 2 \\
\hline$\left\langle\left[\mathrm{Y}_{\mathrm{II}} / \mathrm{Fe} \mathrm{II}\right]\right\rangle$ & -0.13 & -0.04 & -0.23 & 0.19 & 0.02 & 0.16 & -0.04 & 0.18 & 0.08 \\
\hline$\sigma(\log \epsilon(\mathrm{Y}$ II $))$ & 0.04 & 0.04 & 0.04 & 0.06 & 0.11 & 0.09 & 0.09 & 0.04 & 0.03 \\
\hline No. of Lines & 7 & 7 & 6 & 6 & 2 & 4 & 10 & 2 & 3 \\
\hline$\langle[\mathrm{Zr}$ II $/ \mathrm{Fe}$ II $]\rangle$ & 0.21 & 0.27 & 0.14 & 0.53 & 0.35 & 0.38 & 0.35 & $\ldots$ & 0.71 \\
\hline$\sigma(\log \epsilon(\mathrm{Zr}$ II $))$ & 0.06 & 0.12 & 0.17 & 0.16 & $\ldots$ & 0.14 & 0.02 & $\ldots$ & 0.00 \\
\hline No. of Lines & 4 & 4 & 4 & 4 & 1 & 2 & 5 & 0 & 2 \\
\hline$\langle[\mathrm{Ba}$ II $/ \mathrm{Fe}$ II $]\rangle$ & 0.20 & 0.23 & 0.01 & 0.40 & -0.14 & 0.21 & 0.43 & 0.14 & -0.03 \\
\hline$\sigma(\log \epsilon(\mathrm{Ba} \mathrm{II}))$ & 0.03 & 0.10 & 0.07 & 0.08 & 0.03 & 0.05 & 0.10 & 0.07 & 0.06 \\
\hline No. of Lines & 4 & 4 & 4 & 5 & 3 & 5 & 6 & 3 & 3 \\
\hline$\langle[\mathrm{La}$ II $/ \mathrm{Fe}$ II $]\rangle$ & 0.16 & 0.42 & -0.04 & 0.60 & -0.01 & 0.58 & 0.38 & & 0.60 \\
\hline$\sigma(\log \epsilon($ La II $))$ & 0.08 & 0.09 & 0.09 & 0.07 & $\ldots$ & 0.10 & 0.08 & $\ldots$ & 0.14 \\
\hline No. of Lines & 9 & 9 & 9 & 8 & 1 & 6 & 11 & 1 & 2 \\
\hline$\left\langle\left[\mathrm{Ce}\right.\right.$ II $\left.\left./ \mathrm{Fe}_{\mathrm{II}}\right]\right\rangle$ & 0.20 & 0.33 & -0.17 & 0.51 & $\ldots$ & $\ldots$ & 0.37 & $\ldots$ & $\ldots$ \\
\hline$\sigma(\log \epsilon(\mathrm{Ce}$ II $))$ & 0.15 & 0.14 & 0.13 & 0.13 & $\ldots$ & $\ldots$ & 0.14 & $\ldots$ & $\ldots$ \\
\hline No. of Lines & 25 & 22 & 11 & 10 & 0 & 0 & 13 & 0 & 0 \\
\hline$\langle[\operatorname{Pr} \mathrm{II} / \mathrm{Fe} \mathrm{II}]\rangle$ & 0.42 & 0.63 & 0.28 & 0.73 & $\ldots$ & 0.81 & 0.69 & $\ldots$ & $\ldots$ \\
\hline$\sigma(\log \epsilon(\operatorname{Pr} I \mathrm{I}))$ & 0.04 & 0.03 & 0.12 & 0.00 & $\ldots$ & $\ldots$ & 0.11 & $\ldots$ & $\ldots$ \\
\hline No. of Lines & 5 & 5 & 5 & 2 & 0 & 1 & 2 & 0 & 0 \\
\hline$\langle[\mathrm{Nd}$ II $/ \mathrm{Fe}$ II $]\rangle$ & 0.47 & 0.68 & 0.23 & 0.58 & $\ldots$ & $\ldots$ & $\ldots$ & $\ldots$ & $\ldots$ \\
\hline$\sigma(\log \epsilon(\mathrm{Nd} I \mathrm{I}))$ & 0.10 & 0.11 & 0.14 & 0.10 & $\ldots$ & $\ldots$ & $\ldots$ & $\ldots$ & $\ldots$ \\
\hline No. of Lines & 27 & 25 & 22 & 2 & 0 & 0 & 0 & 0 & 0 \\
\hline$\langle[\mathrm{Sm}$ II $/ \mathrm{Fe}$ II $]\rangle$ & 0.50 & 0.77 & 0.29 & 0.61 & $\ldots$ & $\ldots$ & $\ldots$ & $\ldots$ & $\ldots$ \\
\hline$\sigma(\log \epsilon(\operatorname{Sm~II~}))$ & 0.08 & 0.08 & 0.10 & 0.13 & $\ldots$ & $\ldots$ & $\ldots$ & $\ldots$ & $\ldots$ \\
\hline No. of Lines & 20 & 20 & 15 & 3 & 0 & 0 & 0 & 0 & 0 \\
\hline$\langle[\mathrm{Eu} \mathrm{II} / \mathrm{Fe} \mathrm{II}]\rangle$ & 0.50 & 0.83 & 0.26 & 0.90 & 0.72 & 0.85 & 0.69 & 1.18 & 0.96 \\
\hline$\sigma(\log \epsilon($ Eu II $))$ & 0.12 & 0.11 & 0.11 & 0.03 & 0.04 & 0.09 & 0.07 & 0.07 & 0.18 \\
\hline No. of Lines & 7 & 6 & 7 & 5 & 2 & 5 & 5 & 2 & 2 \\
\hline$\langle[\mathrm{Gd} \mathrm{II} / \mathrm{Fe} \mathrm{II}]\rangle$ & 0.55 & 0.77 & 0.25 & 0.69 & $\ldots$ & $\ldots$ & 0.67 & $\ldots$ & $\ldots$ \\
\hline$\sigma(\log \epsilon(\mathrm{Gd} I \mathrm{I}))$ & 0.08 & 0.07 & 0.03 & $\ldots$ & $\ldots$ & $\ldots$ & $\ldots$ & $\ldots$ & $\ldots$ \\
\hline No. of Lines & 8 & 9 & 3 & 1 & 0 & 0 & 1 & 0 & 0 \\
\hline$\langle[\mathrm{Tb}$ II $/ \mathrm{Fe} \mathrm{II}]\rangle$ & 0.56 & 0.67 & 0.42 & $\ldots$ & $\ldots$ & $\ldots$ & 0.96 & $\ldots$ & $\ldots$ \\
\hline$\sigma(\log \epsilon(\mathrm{Tb}$ II $))$ & $\ldots$ & $\ldots$ & $\ldots$ & $\ldots$ & $\ldots$ & $\ldots$ & $\ldots$ & $\ldots$ & $\ldots$ \\
\hline No. of Lines & 1 & 1 & 1 & 0 & 0 & 0 & 1 & 0 & 0 \\
\hline$\langle[$ Dy II $/ \mathrm{Fe}$ II $]\rangle$ & 0.38 & 0.58 & 0.36 & 1.13 & $\ldots$ & $\ldots$ & 0.75 & $\ldots$ & $\ldots$ \\
\hline$\sigma(\log \epsilon($ Dy II $))$ & 0.15 & 0.03 & 0.07 & 0.08 & $\ldots$ & $\ldots$ & 0.11 & $\ldots$ & $\ldots$ \\
\hline No. of Lines & 4 & 2 & 4 & 3 & 0 & 0 & 3 & 0 & 0 \\
\hline$\left\langle\left[\mathrm{Ho}_{\mathrm{II}} / \mathrm{Fe} \mathrm{II}\right]\right\rangle$ & 0.45 & 0.67 & 0.26 & 1.09 & $\ldots$ & 1.35 & 0.81 & $\ldots$ & $\ldots$ \\
\hline$\sigma(\log \epsilon($ Ho II $))$ & 0.05 & 0.06 & 0.10 & 0.10 & $\ldots$ & $\ldots$ & 0.05 & $\ldots$ & $\ldots$ \\
\hline No. of Lines & 3 & 4 & 3 & 3 & 0 & 1 & 4 & 0 & 0 \\
\hline$\langle[\mathrm{Er}$ II $/ \mathrm{Fe}$ II $]\rangle$ & 0.48 & 0.67 & 0.18 & 0.88 & $\ldots$ & 0.73 & 0.58 & $\ldots$ & $\ldots$ \\
\hline$\sigma(\log \epsilon(\operatorname{Er} I I))$ & 0.15 & 0.15 & 0.06 & 0.03 & $\ldots$ & 0.14 & 0.05 & $\ldots$ & $\ldots$ \\
\hline No. of Lines & 4 & 4 & 3 & 4 & 0 & 2 & 3 & 0 & 0 \\
\hline$\left\langle\left[\mathrm{Tm}_{\mathrm{II}} / \mathrm{Fe} \mathrm{II}\right]\right\rangle$ & 0.40 & 0.66 & 0.26 & $\ldots$ & $\ldots$ & $\ldots$ & 0.85 & $\ldots$ & $\ldots$ \\
\hline$\sigma(\log \epsilon(\operatorname{Tm} \mathrm{II}))$ & 0.09 & 0.20 & 0.07 & $\ldots$ & $\ldots$ & $\ldots$ & $\ldots$ & $\ldots$ & $\ldots$ \\
\hline No. of Lines & 3 & 3 & 2 & 0 & 0 & 0 & 1 & 0 & 0 \\
\hline$\langle[\mathrm{Yb}$ II $/ \mathrm{Fe} \mathrm{II}]\rangle$ & 0.23 & 0.64 & 0.09 & 0.93 & 1.29 & 0.71 & $\ldots$ & 0.89 & 0.71 \\
\hline$\sigma(\log \epsilon(\mathrm{Yb} \mathrm{II}))$ & $\ldots$ & $\ldots$ & $\ldots$ & $\ldots$ & $\ldots$ & $\ldots$ & $\ldots$ & $\ldots$ & $\ldots$ \\
\hline No. of Lines & 1 & 1 & 1 & 1 & 1 & 1 & 0 & 1 & 1 \\
\hline$\langle[\mathrm{Hf}$ II $/ \mathrm{Fe}$ II $]>$ & 0.21 & 0.52 & -0.08 & $\ldots$ & $\ldots$ & $\ldots$ & $\ldots$ & $\ldots$ & $\ldots$ \\
\hline$\sigma(\log \epsilon(\mathrm{Hf}$ II $))$ & $\ldots$ & $\ldots$ & $\ldots$ & $\ldots$ & $\ldots$ & $\ldots$ & $\ldots$ & $\ldots$ & $\ldots$ \\
\hline No. of Lines & 1 & 1 & 1 & 0 & 0 & 0 & 0 & 0 & 0 \\
\hline$\langle[\mathrm{Os} \mathrm{I} / \mathrm{Fe} \mathrm{I}]\rangle$ & $\ldots$ & $\ldots$ & $\ldots$ & $\ldots$ & $\ldots$ & $\ldots$ & 2.00 & $\ldots$ & $\ldots$ \\
\hline$\sigma(\log \epsilon(\mathrm{Os} \mathrm{I}))$ & $\ldots$ & $\ldots$ & $\ldots$ & $\ldots$ & $\ldots$ & $\ldots$ & 0.35 & $\ldots$ & $\ldots$ \\
\hline No. of Lines & 0 & 0 & 0 & 0 & 0 & 0 & 2 & 0 & 0 \\
\hline$\langle[\operatorname{Ir} \mathrm{I} / \mathrm{Fe} \mathrm{I}]\rangle$ & $\ldots$ & $\ldots$ & $\ldots$ & $\ldots$ & $\ldots$ & $\ldots$ & 1.35 & $\ldots$ & $\ldots$ \\
\hline$\sigma(\log \epsilon(\operatorname{Ir} \mathrm{I}))$ & $\ldots$ & $\ldots$ & $\ldots$ & $\ldots$ & $\ldots$ & $\ldots$ & $\ldots$ & $\ldots$ & $\ldots$ \\
\hline No. of Lines & 0 & 0 & 0 & 0 & 0 & 0 & 1 & 0 & 0 \\
\hline$\langle[\mathrm{Pb} \mathrm{I} / \mathrm{Fe} \mathrm{I}]\rangle$ & $\lesssim 0.44$ & $\lesssim 0.35$ & $\lesssim 0.38$ & $\ldots$ & $\ldots$ & $\ldots$ & $\ldots$ & $\ldots$ & $\ldots$ \\
\hline$\sigma(\log \epsilon(\mathrm{Pb} \mathrm{I}))$ & $\ldots$ & $\ldots$ & $\sim \ldots$ & $\ldots$ & $\ldots$ & $\ldots$ & $\ldots$ & $\ldots$ & $\ldots$ \\
\hline No. of Lines & 1 & 1 & 1 & 0 & 0 & 0 & 0 & 0 & 0 \\
\hline langle $[\mathrm{Th} \mathrm{II} / \mathrm{Fe} \mathrm{II}]>$ & 0.46 & 0.67 & 0.32 & $\ldots$ & $\ldots$ & $\ldots$ & $\ldots$ & $\ldots$ & $\ldots$ \\
\hline$\sigma(\log \epsilon($ Th II $))$ & $\ldots$ & $\ldots$ & $\ldots$ & $\ldots$ & $\ldots$ & $\ldots$ & $\ldots$ & $\ldots$ & $\ldots$ \\
\hline No. of Lines & 1 & 1 & 1 & 0 & 0 & 0 & 0 & 0 & 0 \\
\hline
\end{tabular}


Table 5

Solar Photospheric Abundances of the Elements Analyzed in the Current Study

\begin{tabular}{|c|c|c|c|c|}
\hline Element & $\mathrm{Z}$ & $\log \left(\epsilon_{\text {Elem }}\right) \odot$ & Reference & Methodology \\
\hline $\mathrm{H}$ & 1 & 12.00 & $\mathrm{~N} / \mathrm{A}$ & \\
\hline $\mathrm{C}$ & 6 & $8.43 \pm 0.05$ & Asplund et al. (2009) & 3-D; LTE \\
\hline $\mathrm{O}$ & 8 & $8.71 \pm 0.05$ & Scott et al. (2009) & 3-D; LTE \\
\hline $\mathrm{Na}$ & 11 & $6.17 \pm 0.04$ & Asplund et al. (2005) & 3-D; LTE \\
\hline $\mathrm{Mg}$ & 12 & $7.53 \pm 0.09$ & Asplund et al. (2005) & 3-D; LTE \\
\hline $\mathrm{Al}$ & 13 & $6.37 \pm 0.06$ & Asplund et al. (2005) & 3-D; LTE \\
\hline $\mathrm{Si}$ & 14 & $7.51 \pm 0.04$ & Asplund et al. (2005) & 3-D; LTE \\
\hline $\mathrm{Ca}$ & 20 & $6.31 \pm 0.04$ & Asplund et al. (2005) & 3-D; LTE \\
\hline Sc & 21 & $3.15 \pm 0.04$ & Asplund et al. (2009); Grevesse et al. (2010) & 3-D; LTE \\
\hline $\mathrm{Ti}$ & 22 & $4.95 \pm 0.05$ & Asplund et al. (2009); Grevesse et al. (2010) & 3-D; LTE \\
\hline V & 23 & $3.93 \pm 0.08$ & Asplund et al. (2009); Grevesse et al. (2010) & 3-D; LTE \\
\hline $\mathrm{Cr}$ & 24 & $5.64 \pm 0.02$ & Sobeck et al. (2007) & 1-D; LTE \\
\hline $\mathrm{Mn}$ & 25 & $5.43 \pm 0.04$ & Asplund et al. (2009); Grevesse et al. (2010) & 3-D; LTE \\
\hline $\mathrm{Fe}$ & 26 & $7.52 \pm 0.08$ & Sneden et al. (1991) & 1-D; LTE \\
\hline Co & 27 & $4.99 \pm 0.08$ & Asplund et al. (2009); Grevesse et al. (2010) & 3-D; LTE \\
\hline $\mathrm{Ni}$ & 28 & $6.17 \pm 0.02$ & Scott et al. (2009) & 3-D; LTE \\
\hline $\mathrm{Cu}$ & 29 & $4.19 \pm 0.04$ & Asplund et al. (2009); Grevesse et al. (2010) & 3-D; LTE \\
\hline $\mathrm{Zn}$ & 30 & $4.60 \pm 0.03$ & Biémont \& Godefroid (1980) & 1-D; LTE \\
\hline $\mathrm{Sr}$ & 38 & $2.92 \pm 0.05$ & Barklem \& O’Mara (2000) & 1-D; LTE \\
\hline $\mathrm{Y}$ & 39 & $2.21 \pm 0.05$ & Asplund et al. (2009); Grevesse et al. (2010) & 3-D; LTE \\
\hline $\mathrm{Zr}$ & 40 & $2.58 \pm 0.04$ & Asplund et al. (2009); Grevesse et al. (2010) & 3-D; LTE \\
\hline $\mathrm{Ba}$ & 56 & $2.17 \pm 0.09$ & Asplund et al. (2009); Grevesse et al. (2010) & 3-D; LTE \\
\hline $\mathrm{La}$ & 57 & $1.13 \pm 0.03$ & Lawler et al. (2001a) & 1-D; LTE \\
\hline $\mathrm{Ce}$ & 58 & $1.61 \pm 0.01$ & Lawler et al. (2009) & 1-D; LTE \\
\hline $\operatorname{Pr}$ & 59 & $0.76 \pm 0.02$ & Sneden et al. (2009) & 1-D; LTE \\
\hline $\mathrm{Nd}$ & 60 & $1.45 \pm 0.01$ & Den Hartog et al. (2003) & 1-D; LTE \\
\hline $\mathrm{Sm}$ & 62 & $1.00 \pm 0.01$ & Lawler et al. (2006) & 1-D; LTE \\
\hline $\mathrm{Eu}$ & 63 & $0.52 \pm 0.01$ & Lawler et al. (2001c) & 1-D; LTE \\
\hline $\mathrm{Gd}$ & 64 & $1.11 \pm 0.01$ & Den Hartog et al. (2006) & 1-D; LTE \\
\hline $\mathrm{Tb}$ & 65 & $0.28 \pm 0.3$ & Lawler et al. (2001b) & 1-D; LTE \\
\hline Dy & 66 & $1.13 \pm 0.02$ & Sneden et al. (2009) & 1-D; LTE \\
\hline Ho & 67 & $0.51 \pm 0.10$ & Lawler et al. (2004) & 1-D; LTE \\
\hline $\mathrm{Er}$ & 68 & $0.96 \pm 0.03$ & Lawler et al. (2008) & 1-D; LTE \\
\hline $\mathrm{Tm}$ & 69 & $0.14 \pm 0.02$ & Sneden et al. (2009) & 1-D; LTE \\
\hline $\mathrm{Yb}$ & 70 & $0.86 \pm 0.10$ & Sneden et al. (2009) & 1-D; LTE \\
\hline Hf & 72 & $0.88 \pm 0.08$ & Lawler et al. (2007) & 1-D; LTE \\
\hline Os & 76 & $1.25 \pm 0.11$ & Quinet et al. (2006) & 1-D; LTE \\
\hline $\mathrm{Ir}$ & 77 & $1.38 \pm 0.05$ & Youssef \& Khalil (1988) & 1-D; LTE \\
\hline $\mathrm{Pb}$ & 82 & $1.75 \pm 0.10$ & Asplund et al. (2009); Grevesse et al. (2010) & 3-D; LTE \\
\hline $\mathrm{Th}$ & 90 & $0.02 \pm 0.10$ & Asplund et al. (2009); Grevesse et al. (2010) & 3-D; LTE \\
\hline
\end{tabular}

exhibit abundance trends similar to those of the RGB objects. The expected anti-correlations in the proton-capture elements (e.g., $\mathrm{Na}-\mathrm{O}$ and $\mathrm{Mg}-\mathrm{Al}$ ) are seen. The greatest abundance variation with regard to the entire M15 data set is found for the neutron-capture elements. Indeed, the star-to-star spread for the majority of $n$-capture abundances is demonstrable for all M15 targets and is not likely due to internal errors.

Inspection of Table 4 data indicates that RHB stars generally have higher $r$-process element abundances than RGB stars (on average $\left.\Delta[\mathrm{Elem} / \mathrm{Fe}]_{\mathrm{RHB}-\mathrm{RGB}} \approx 0.3 \mathrm{dex}\right)$. A sizeable portion of the discrepancy is attributable to the difference in the iron abundances as $\langle[\mathrm{Fe} / \mathrm{H}]\rangle_{\mathrm{RHB}}$ is approximately $0.12 \mathrm{dex}$ lower than $\langle[\mathrm{Fe} / \mathrm{H}]\rangle_{\mathrm{RGB}}$. The remaining offset is most likely a consequence of the small number of targets coupled with a serious selection effect. The original sample of RHB stars from Preston et al. (2006) was chosen as a random set of objects with colors and magnitudes representative of the red end of the HB. These objects were selected without prior knowledge of the heavy element abundances. On the other hand, the three RGB stars from Sneden et al. (2000a) were particularly chosen as representing the highest and lowest abundances of the $r$-process as predetermined in the 17 star sample of Sneden et al. (1997).

\subsection{Light Element Abundances}

The finalized set of light element abundances include: $\mathrm{C}, \mathrm{O}$, $\mathrm{Mg}, \mathrm{Al}, \mathrm{Si}, \mathrm{Ca}$, and $\mathrm{Sc}_{\mathrm{I} / \mathrm{II}}$. In general, an enhancement of these element abundances relative to solar is seen in the entire M15 data set.

An underabundance of carbon was found in one RHB and three RGB targets of M15 based on the measurement of $\mathrm{CH}$ spectral features. As the forbidden $\mathrm{O}_{\mathrm{I}}$ lines were detectable only in RGB stars, the average abundance ratio for M15 is $\langle[\mathrm{O} / \mathrm{Fe}]\rangle_{\mathrm{RGB}}=+0.75$. This value is substantially larger than that found by Sneden et al. (1997). A portion of the discrepancy is due to the approximate 0.15 dex difference in the $\left\langle\left[\mathrm{Fe}_{\mathrm{I}} / \mathrm{H}\right]\right\rangle$ values between the two investigations. The remainder of departure may be attributed to the adoption of different solar photospheric oxygen values: the current study employs $\log \epsilon(\mathrm{O})_{\odot}=8.71$ (Scott et al. 2009), while Sneden et al. use $\log \epsilon(\mathrm{O})_{\odot}=8.93$ (Anders \& Grevesse 1989). 
For the determination of the sodium abundance, the current study relies solely upon the $\mathrm{D}_{1}$ resonance transitions. Table 4 lists the spuriously large spreads in the $\mathrm{Na}$ abundance for both the RGB and RHB groups. The $\mathrm{Na}_{1}$ lines are affected by the non-LTE phenomenon of resonance scattering (Asplund 2005b; Andrievsky et al. 2007), which MOOG does not take into account. Also, Sneden et al. (2000b) made note of the relative strength and line profile distortions associated with these transitions and chose to discard the $[\mathrm{Na} / \mathrm{Fe}]$ values for stars with $T_{\text {eff }}>5000 \mathrm{~K}$. Consequently, the sodium results from the current study are given little weight and are not plotted in Figure 3.

Aluminum is remarkable in its discordance: $\left\langle\left[\mathrm{Al}_{\mathrm{I}} / \mathrm{Fe}_{\mathrm{I}}\right]\right\rangle=$ 0.37 for one RHB and three RGB targets, whereas $\left\langle\left[\mathrm{Al}_{\mathrm{I}} / \mathrm{Fe}_{\mathrm{I}}\right]\right\rangle=-0.43$ for five RHB stars. Now, the relative aluminum abundances for the RHB stars match well with the values found by Preston et al. (2006). Similarly, the Al abundances from the current analysis agree favorably with the RGB data from Sneden et al. (1997). Though relatively strong transitions are employed in the abundance derivation, the convergence upon two distinct $[\mathrm{Al} / \mathrm{Fe}]$ values is nontrivial and could merit further exploration.

A decidedly consistent $\mathrm{Ca}$ abundance ratio is found for the RGB sample: $\left\langle\left[\mathrm{Ca}_{\mathrm{I}} / \mathrm{Fe}_{\mathrm{I}}\right]\right\rangle_{\mathrm{RGB}}=0.29$; and also for the RHB sample: $\left\langle\left[\mathrm{Ca}_{\mathrm{I}} / \mathrm{Fe}_{\mathrm{I}}\right]\right\rangle_{\mathrm{RHB}}=0.53$. After consideration of the iron abundance offset, the RHB stars still report slightly higher calcium abundances than the RGB stars. Overall, a distinct overabundance of $\mathrm{Ca}$ relative to solar is present in the M15 cluster. Note that the $\mathrm{Sc}_{\mathrm{I}}$ abundance determination was done for only one M15 star (and gives a rather aberrant result compared to the $\mathrm{Sc}_{\mathrm{II}}$ abundance data from the other M15 targets).

\subsection{Iron-peak Element Abundances}

The list of finalized Fe-peak element abundances consists of $\mathrm{Ti}_{\mathrm{I} / \mathrm{II}}, \mathrm{V}_{\mathrm{I} / \mathrm{II}}, \mathrm{Cr}_{\mathrm{I} / \mathrm{II}}, \mathrm{Mn}, \mathrm{Co}$, and Ni. Due to RGB spectral crowding issues, derivations of $\left[\mathrm{Ni}_{\mathrm{I}} / \mathrm{Fe}_{\mathrm{I}}\right]$ ratios are performed only for RHB stars.

Achievement of ionization equilibrium did not occur for any of the perspective species: $\mathrm{Ti}_{\mathrm{I} / \mathrm{II}}, \mathrm{V}_{\mathrm{I} / \mathrm{II}}$, or $\mathrm{Cr}_{\mathrm{I} / \mathrm{II}}$. In consideration of the entire M15 data set, the best agreement between neutral and singly ionized species arises for titanium, with all $\langle[\mathrm{Ti} / \mathrm{Fe}]\rangle$ ratios being supersolar. The $\mathrm{V}_{\mathrm{II}}$ relative abundances compare well with one another for the RGB and RHB targets (comparison for $\mathrm{V}_{\mathrm{I}}$ is not possible as there are no RHB data for this species). Both of the RGB and RHB $\left\langle\left[\mathrm{Cr}_{I} /\right.\right.$ $\left.\left.\mathrm{Fe}_{\mathrm{I}}\right]\right\rangle$ ratios are underabundant with respect to solar and the neutral chromium values match almost exactly with one another (after accounting for the $[\mathrm{Fe} / \mathrm{H}]$ offset). On the other hand, the worst agreement is found for $\mathrm{Cr}_{\mathrm{I} / \mathrm{II}}$ in RHB stars with $\Delta(\mathrm{II}-\mathrm{I})=$ 0.47 .

Subsolar values with minimal scatter were found for the $\left\langle\left[\mathrm{Mn}_{\mathrm{I}} / \mathrm{Fe}_{\mathrm{I}}\right]\right\rangle$ ratios in both the RGB and RHB stellar groups. However, in comparison to RGB stars, manganese appears to be substantially more deficient in RHB targets. The discrepancy may be attributed to both the RGB/RHB iron abundance disparity as well as the employment of the $\mathrm{Mn}_{\mathrm{I}}$ resonance transition at $4034.5 \AA$ for the RHB abundance determination. In particular, J. S. Sobeck et al. (2011, in preparation) have demonstrated that the manganese resonance triplet (4030.7, 4033.1, and 4034.5 $\AA$ ) fails to be a reliable indicator of abundance. Consequently, the RHB abundance results for $\mathrm{Mn}_{\mathrm{I}}$ are given little weight and are not plotted in Figure 4.

\subsection{Light and Intermediate n-capture Element Abundances}

Finalized abundances for the light and intermediate $n$-capture elements include $\mathrm{Cu}, \mathrm{Zn}, \mathrm{Sr}, \mathrm{Y}, \mathrm{Zr}, \mathrm{Ba}, \mathrm{La}, \mathrm{Ce}$, and Pr. In general, the RGB element abundance ratios are slightly deficient with respect to the RHB values. Also, enhancement with respect to solar is consistently seen in all M15 targets for the elements Ce and Pr.

An extremely underabundant copper abundance relative to solar was found in the RGB stars: $\left\langle\left[\mathrm{Cu}_{\mathrm{I}} / \mathrm{Fe}_{\mathrm{I}}\right]\right\rangle_{\mathrm{RGB}}=-0.91$. A similar derivation could not take place in the RGB targets as the $\mathrm{Cu}_{\mathrm{I}}$ transitions were too weak. A large divergence between RGB and RHB stellar abundances exists for zinc. Detection of the $\mathrm{Zn}$ transitions was possible in only one RHB target, which could perhaps account for some of the discrepancy.

For the entire M15 data set, $\mathrm{Y}_{\mathrm{II}}$ exhibits lower relative abundance ratios in juxtaposition to both $\mathrm{Sr}_{\mathrm{II}}$ and $\mathrm{Zr}_{\mathrm{II}}$. With regard to the three average elemental abundances (of Sr, Y, Zr), moderate departures between the RGB and RHB groups are seen. Also, a large variation in the $\left\langle\left[\mathrm{Sr}_{\mathrm{II}} / \mathrm{Fe}_{\mathrm{II}}\right]\right\rangle$ ratio was found for the members of the RGB group.

Though different sets of lines are employed, the RGB and $\mathrm{RHB}\left\langle\left[\mathrm{Ba}_{\mathrm{II}} / \mathrm{Fe}_{\mathrm{II}}\right]\right\rangle$ ratios are consistent with one another. A portion of the RHB abundance variation is due to the exclusive use of the resonance transitions in the determination (two lowest temperature RHB stars report quite high $\sigma$ values; these strong lines could not be exploited in the RGB analysis). Notably for this element group, the greatest star-to-star abundance scatter was found for lanthanum: $\Delta_{\mathrm{RGB}}=0.46$ and $\Delta_{\mathrm{RHB}}=$ 0.61 (excluding the one RHB outlier). The relative cerium abundances also exhibit a wide spread in the RGB sample.

\subsection{Heavy n-capture Element Abundances}

The list of finalized $\langle[\mathrm{El} / \mathrm{Fe}]\rangle$ ratios for the heavy $n$-capture elements is as follows: $\mathrm{Nd}, \mathrm{Sm}, \mathrm{Eu}, \mathrm{Gd}, \mathrm{Tb}, \mathrm{Dy}, \mathrm{Ho}, \mathrm{Er}, \mathrm{Tm}, \mathrm{Yb}$, $\mathrm{Hf}, \mathrm{Os}, \mathrm{Ir}, \mathrm{Pb}$, and Th. All of these element abundance ratios are enriched with regard to the solar values. As shown in Figure 6, a larger abundance spread is found for this group in comparison to the other element groups.

Note that as $T_{\text {eff }}$ increases, the strength of the heavy element transitions rapidly decreases and as a consequence, the use of these lines for abundance determinations in the warmest stars becomes unfeasible. It was possible to obtain robust abundances for $\mathrm{Nd}, \mathrm{Sm}, \mathrm{Tb}$, and $\mathrm{Tm}$ in a single RHB target. On the other hand, abundance extractions for the species Os and Ir were done only in RHB stars (measurements of these element transitions were attainable as less spectral crowding occurs in these stars). Nonetheless, minimal line-to-line scatter is seen for the bulk of RGB and RHB $n$-capture abundances.

A rigorous determination of the europium relative abundance was performed for all M15 stars: $\left\langle\left[\mathrm{Eu}_{\mathrm{II}} / \mathrm{Fe}_{\mathrm{II}}\right]\right\rangle_{\mathrm{RGB}}=0.53$ and $\left\langle\left[\mathrm{Eu}_{\mathrm{II}} / \mathrm{Fe}_{\mathrm{II}}\right]\right\rangle_{\mathrm{RHB}}=0.88$. Despite the iron abundance offset, the largest departure between the two stellar groups is found for the element $\mathrm{Ho}_{\mathrm{II}}$. Further, the greatest star-to-star scatter in the heavy $n$-capture elements is seen for the $\left[\mathrm{Yb}_{\mathrm{II}} / \mathrm{Fe}_{\mathrm{II}}\right]$ ratio: $\Delta_{\mathrm{RGB}}=0.55$ and $\Delta_{\mathrm{RHB}}=0.58$.

\subsection{Comparison with Previous CTG Efforts and Otsuki et al. (2006)}

These new abundance results are now compared to those from the four prior CTG publications. For the majority of elements, the current data are in accord with the findings of Sneden et al. (1997, 2000a) and Preston et al. (2006). In this effort, abundance 
derivations are performed for 13 new species: $\mathrm{Sc}_{\mathrm{I}}, \mathrm{V}_{\mathrm{II}}, \mathrm{Cu}_{\mathrm{I}}, \mathrm{Pr}_{\mathrm{II}}$, $\mathrm{Tb}_{\mathrm{II}}, \mathrm{Ho}_{\mathrm{II}}, \mathrm{Er}_{\mathrm{II}}, \mathrm{Tm}_{\mathrm{II}}, \mathrm{Yb}_{\mathrm{II}}, \mathrm{Hf}_{\mathrm{II}}, \mathrm{Os}_{\mathrm{I}}, \mathrm{Ir}_{\mathrm{I}}$, and $\mathrm{Pb}_{\mathrm{II}}$. For elements re-analyzed in the current study, the abundance data have been improved with the use of higher quality atomic data, additional transitions, and a revised version of the MOOG program. A few large discrepancies in the $[\mathrm{El} / \mathrm{Fe}]$ ratios do occur between the current study and the previous M15 efforts. These departures can be attributed to the employment of different $[\mathrm{Fe} / \mathrm{H}]$ and solar photospheric values as well as the updated MOOG code. Accordingly, the results from the current analysis supersede those from the earlier CTG papers.

As in Sneden et al. (1997), the abundance behavior of the proton-capture elements appears to be decoupled from that of the neutron-capture elements. Notably for M15, significant spread in the abundances was confirmed for both $\mathrm{Ba}$ and $\mathrm{Eu}$. The scatter of $\Delta \log \epsilon(\mathrm{Ba})=0.48$ and $\Delta \log \epsilon(\mathrm{Eu})=0.90$ from the current effort is in line with that of $\Delta \log \epsilon(\mathrm{Ba})=0.60$ and $\Delta \log \epsilon(\mathrm{Eu})=0.73$ from Sneden et al. (1997).

Comparison of the findings from the current study to those from Otsuki et al. (2006) has also been done and will be limited to the only star that the two investigations have in common, K462. Due to differences in the $\langle[\mathrm{Fe} / \mathrm{H}]\rangle$ values, the $\log (\epsilon)$ data of the two analyses are compared. The model atmospheric parameters for K462 differ somewhat between the current effort $\left(T_{\text {eff }} / \log g / v_{\mathrm{t}}=4400 / 0.30 / 2.00\right)$ and Otsuki et al. $\left(T_{\text {eff }} / \log g / v_{\mathrm{t}}=4225 / 0.50 / 2.25\right)$. However, the agreement in the abundances for the elements $\mathrm{Y}, \mathrm{Zr}, \mathrm{Ba}, \mathrm{La}$, and $\mathrm{Eu}$ is rather good between the two studies, with the exact differences ranging: $0.01 \leqslant \mid \Delta$ (Otsuki - Current) $\mid \leqslant 0.16$. The largest disparity occurs for $\mathrm{Sr}$, with both analyses employing the resonance transitions. As mentioned previously, these lines are not the most rigorous probes of abundance.

\subsection{General Relationship of Ba, La, and Eu Abundances}

Sneden et al. (1997) claimed to have found a binary distribution in a plot of $[\mathrm{Ba} / \mathrm{Fe}]$ versus $[\mathrm{Eu} / \mathrm{Fe}]$, with eight stars exhibiting relative $\mathrm{Ba}$ and $\mathrm{Eu}$ abundances approximately 0.35 dex smaller than the remainder of the M15 data set. To re-examine their assertion, Figure 7 is generated, which plots $[(\mathrm{Ba}, \mathrm{La}) / \mathrm{H}]$ as a function of $[\mathrm{Eu} / \mathrm{H}]$ for the entire data sample of the current study. It also displays the re-derived/re-scaled $\mathrm{Ba}, \mathrm{La}$, and $\mathrm{Eu}$ abundances for all of the giants from the Sneden et al. (1997) publication. No decisive offset is evident in either panel of Figure 7. For completeness, the EW data from Otsuki et al. (2006) were also re-analyzed and the abundances were redetermined. Again, no bifurcation was detected in the $\mathrm{Ba}$ and Eu data. ${ }^{13}$

\section{DATA INTERPRETATION AND ANALYSIS}

A significant amount of $r$-process enrichment has occurred in the M15 GC. Figure 8 plots the average $\log \epsilon$ values of the $n$-capture elements (with $39 \leqslant Z \leqslant 70$ ) for three RGB stars (K341, K462, K583; signified by red symbols) and three RHB stars (B009, B224, B262; denoted by blue symbols). ${ }^{14}$ The solid black line in this figure indicates the scaled, solar $r$-process prediction as computed by Sneden et al. (2008). All of the element abundances are normalized to the individual stellar $\log \left(\epsilon_{\mathrm{Eu}}\right)$ values $(\mathrm{Eu}$ is assumed to be an indicator of $r$-process

\footnotetext{
13 To avoid duplication, the stars from Ostuki et al. are not plotted as they are a subset of the original sample from the Sneden et al. (1997) study.

14 B028, B412, and B584 are not included in the figure as these stars lack abundances for most of the elements in the specified $Z$ range.
}

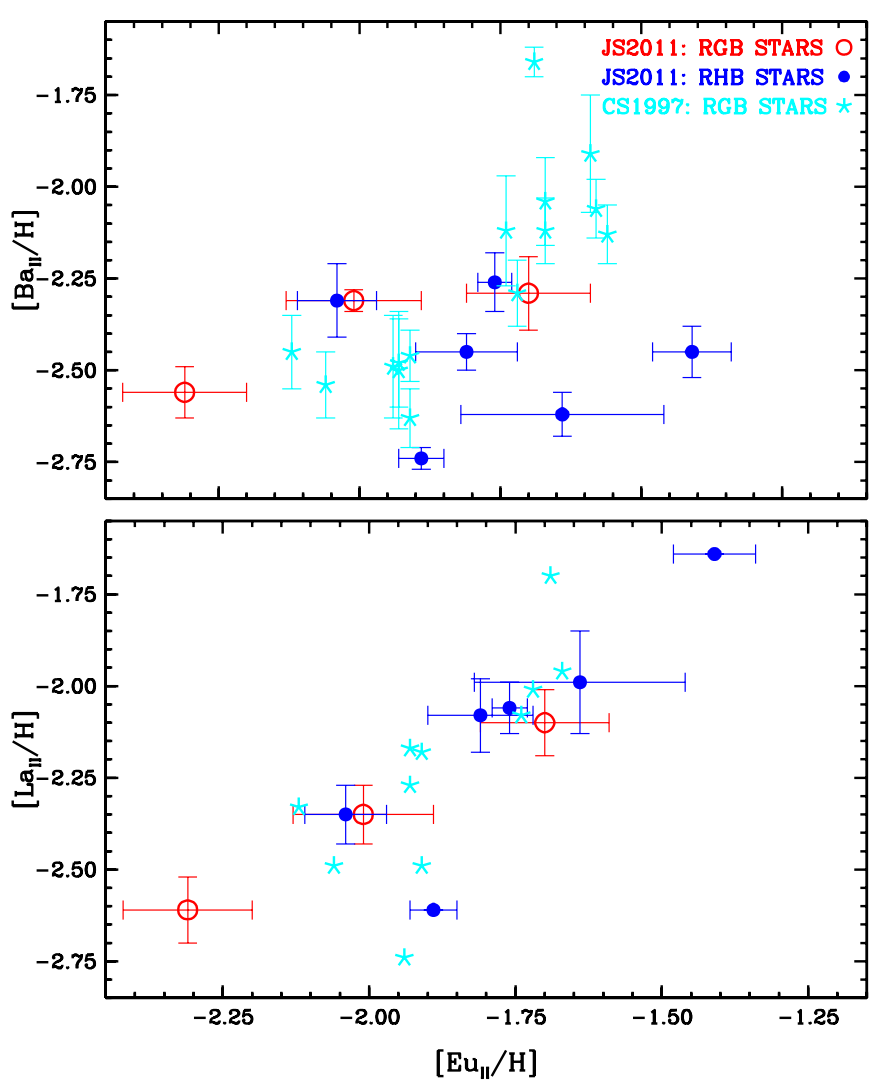

Figure 7. Plots of $[(\mathrm{Ba}, \mathrm{La}) / \mathrm{H}]$ as a function of $[\mathrm{Eu} / \mathrm{H}]$ for the M15 stars of the current study as well as those from the original Sneden et al. (1997) paper. Also shown are the associated error bars for the abundances (these are the standard deviation values given in Table 4). Note that the Eu and La abundance determinations for the 1997 stellar sample are based upon one transition only (and consequently, no error bars are plotted). Contrary to Sneden et al., no clear evidence of a binary distribution in the abundances is detected.

(A color version of this figure is available in the online journal.)

contribution). For the $n$-capture elements with $Z=64-72$, the RGB stellar abundance values strongly correlate with the solar $r$-process distribution. Similarly for the RHB stars, these abundances match well to the solar $r$-process pattern for most of the elements in the $Z=64-72$ range.

Figure 8 also displays the scaled, solar $s$-process abundance distribution (green, dotted line). The $s$-process predictions are also taken from Sneden et al. and the values are normalized to the solar $\log \left(\epsilon_{\mathrm{Ba}}\right)$ (Ba is considered to be an indicator of $s$-process contribution). As shown for the $Z=64-72$ elements, there is virtually no agreement between the solar $s$-process pattern and either the RGB or the RHB stellar abundances. The $s$-process predictions compare well to the RGB abundances for only two elements: $\mathrm{Ce}$ and $\mathrm{La}$. Thus, it follows that the nucleosynthesis of the heavy neutron-capture elements in M15 was dominated by the $r$-process. In addition, the abundance pattern for the light $n$-capture elements $(\mathrm{Sr}, \mathrm{Y}, \mathrm{Zr}$ ) does not adhere to either a solar $r$-process or $s$-process distribution.

\subsection{Evidence for Additional Nucleosynthetic Mechanisms Beyond the Classical $r$ - and s-process}

To further examine the anomalous light $n$-capture abundances in the M15 cluster, Figures 9 and 10 are generated. For the stars from the current effort and those from Otsuki et al. (2006), these two plots display the abundances of the $n$-capture elements $(\mathrm{Sr}$, $\mathrm{Y}, \mathrm{Zr}$, and $\mathrm{La})$ as a function of the $[\mathrm{Ba} / \mathrm{H}]$ and $[\mathrm{Eu} / \mathrm{H}]$ ratios, 


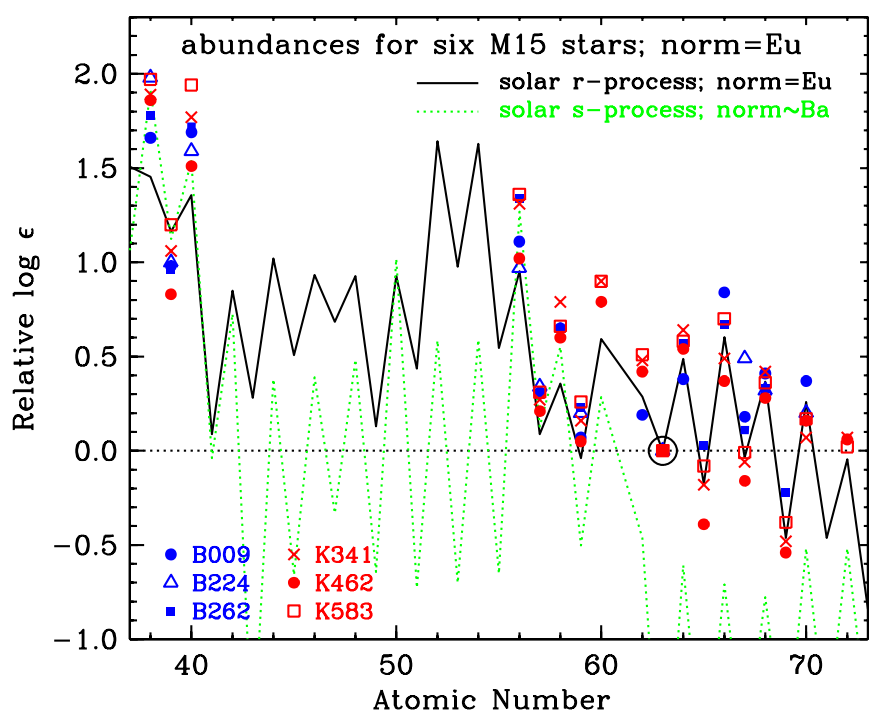

Figure 8. Comparison of the $n$-capture abundances of six M15 targets to the solar system $r$-and $s$-only abundance distributions. The abundances of the three RGB stars (K341, K462, and K583) are signified by red symbols, while the abundances of the three RHB stars (B009, B224, and B262) are designated by blue symbols. The solid black line denotes the $r$-process only abundance pattern, which is scaled to the solar $\log \epsilon(\mathrm{Eu})$, and the dotted green line indicates the $s$-process only abundance pattern, which is scaled to the solar $\log \epsilon(\mathrm{Ba})$ (all predictions are taken from Sneden et al. 2008). The heavy element abundances of the M15 stars compare well to the $r$-process predictions, but not those of the $s$-process. Neither abundance distribution consistently matches the stellar abundances for the elements $\mathrm{Sr}, \mathrm{Y}$, and $\mathrm{Zr}$.

(A color version of this figure is available in the online journal.)

respectively. Moreover, the abundance results from five select field stars, which represent extremes in $r$-process or $s$-process enhancement, are plotted (CS 22892-052: Sneden et al. 2003, 2009; CS 22964-161: Thompson et al. 2008; HD 115444: Westin et al. 2000; Sneden et al. 2009; HD 122563: Cowan et al. 2005; Lai et al. 2007; HD 221170: Ivans et al. 2006; Sneden et al. 2009).

In Figure 9, an anti-correlative trend is seen for Sr, Y, and $\mathrm{Zr}$ with $\mathrm{Ba}$ while no explicit correlative behavior is apparent for La. The correlation coefficient, $r$, is indicated in each panel. Likewise, La and Eu appear un-correlated in Figure 10. The [(Sr, $\mathrm{Y}, \mathrm{Zr}) / \mathrm{Eu}]$ ratios all exhibit anti-correlation with $[\mathrm{Eu} / \mathrm{H}]$ in this figure. As shown, the elements $\mathrm{Sr}, \mathrm{Y}$, and $\mathrm{Zr}$ clearly demonstrate an anti-correlative relationship with both the markers of the $s$ process $(\mathrm{Ba})$ and the $r$-process $(\mathrm{Eu})$.

Figures 9 and 10 collectively imply that the production of the light neutron-capture elements most likely did not transpire via the classical forms of the $s$-process or the $r$-process. This finding is not novel. The abundance survey of halo field stars by Travaglio et al. (2004) previously established the decoupled behavior of the light $n$-capture species to both $\mathrm{Ba}$ and $\mathrm{Eu}$. Further, they postulated that an additional nucleosynthetic process was necessary for the production of these elements ( $\mathrm{Sr}$, $\mathrm{Y}, \mathrm{Zr}$ ) in metal-deficient regimes (coined the Lighter Element Primary Process, LEPP).

The overabundances of $\mathrm{Sr}$ and $\mathrm{Zr}$ (see Figure 8) could have been the result of a small $s$-process contribution to the M15 protocluster environment. To investigate this possibility, an abundance determination is performed for $\mathrm{Pb}$, a definitive main $s$-process product. The upper panel of Figure 11 illustrates the synthetic spectrum fits to the neutral $\mathrm{Pb}$ transition at a wavelength of $4057.8 \AA$ in the M15 giant, K462. An upper limit of $\log \epsilon(\mathrm{Pb}) \lesssim-0.35$ can only be established for this
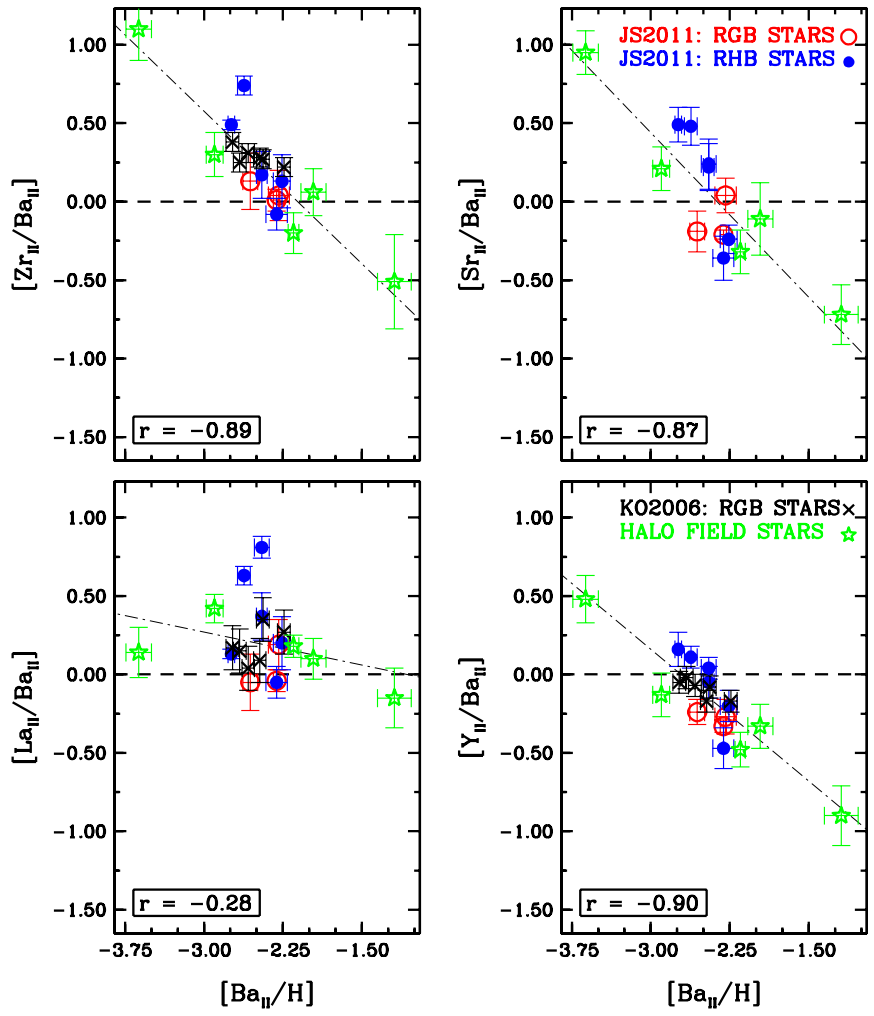

Figure 9. Plot of the relative abundances of the light $n$-capture elements $\mathrm{Sr}, \mathrm{Y}$, and $\mathrm{Zr}$ as function of the traditional $s$-process indicator, Ba. All M15 stars from the current effort are shown. Also displayed are the abundance results from Otsuki et al. (2006) as well as those for a few halo field stars (taken from various literature references; see the text for further details). The correlation coefficient, $\mathbf{r}$, is exhibited in each of the panels and, as seen, Sr, Y, and $\mathrm{Zr}$ demonstrate a clear anti-correlative behavior with $\mathrm{Ba}$ while La does not.

(A color version of this figure is available in the online journal.)

star. For the remaining two RGB targets, upper limits were also determined and, accordingly for all three, the average values of $\log \epsilon(\mathrm{Pb}) \lesssim-0.4$ and $\langle[\mathrm{Pb} / \mathrm{Eu}]\rangle \lesssim-0.15$ were found.

The lower panel of Figure 11 plots $[\mathrm{Pb} / \mathrm{Eu}]$ as a function of $[\mathrm{Eu} / \mathrm{Fe}]$ for the three M15 RGB stars and the five previously employed halo field stars. In a recent paper, Roederer et al. (2010) suggest that detections of $\mathrm{Pb}$ and enhanced $[\mathrm{Pb} / \mathrm{Eu}]$ ratios should be strong indicators of main $s$-process nucleosynthesis. In turn, they contend that non-detections of $\mathrm{Pb}$ and depleted $[\mathrm{Pb} / \mathrm{Eu}]$ ratios should signify the absence of nucleosynthetic input from the main component of the $s$-process (see their paper for further discussion). With the abundances of 161 low-metallicity stars $([\mathrm{Fe} / \mathrm{H}]<-1)$, Roederer et al. empirically determined a threshold value of $[\mathrm{Pb} / \mathrm{Eu}]=+0.3$ for minimum asymptotic giant branch (AGB) contribution. As shown in the figure, the M15 giants lie below this threshold and, accordingly, are likely devoid of main $s$-process input. Thus, in the case of the M15 GC, the light neutron-capture elements presumably originated from an alternate nucleosynthetic process (e.g., $v-p$ process; Fröhlich et al. 2006; high entropy winds; Farouqi et al. 2009).

\subsection{M15 Abundances in Relation to the Halo Field}

The upper panel of Figure 12 displays the evolution of the $[\mathrm{Mg} / \mathrm{Fe}]$ abundance ratio with $[\mathrm{Fe} / \mathrm{H}]$ for all $\mathrm{M} 15$ targets as well as for a sample of hundreds of field stars. For this figure, halo and disk star data have been taken from these surveys: Fulbright (2000), Reddy et al. (2003), Cayrel et al. (2004), 

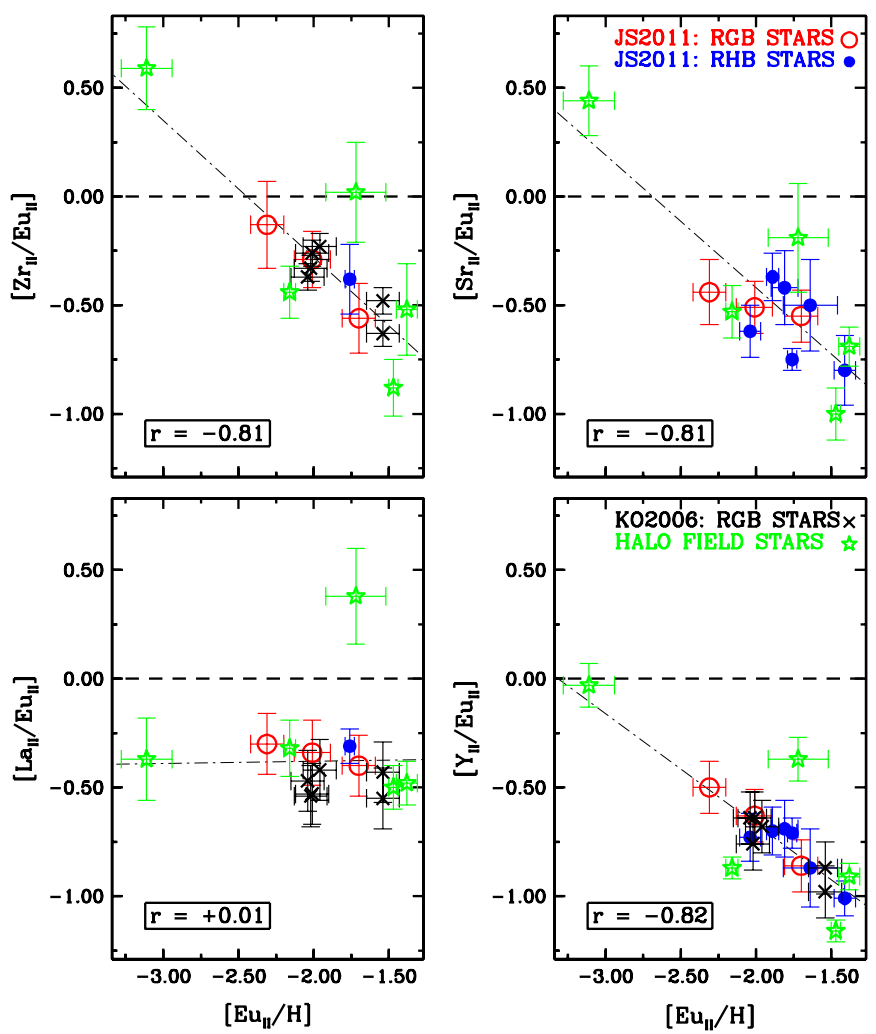

Figure 10. Plot of the relative abundances of the light $n$-capture elements $\mathrm{Sr}$ $\mathrm{Y}$, and $\mathrm{Zr}$ as function of the traditional $r$-process indicator, Eu. All M15 stars from the current effort are shown. Also displayed are the abundance results from Otsuki et al. (2006) as well as those for a few halo field stars (taken from various literature references; see the text for further details). The correlation coefficient, $\mathbf{r}$, is exhibited in each of the panels and, as seen, $\mathrm{Sr}, \mathrm{Y}$, and $\mathrm{Zr}$ demonstrate a clear anti-correlative behavior with Eu while La does not.

(A color version of this figure is available in the online journal.)

Cohen et al. (2004), Simmerer et al. (2004), Barklem et al. (2005), Reddy et al. (2006), François et al. (2007), and Lai et al. (2008). As shown, the scatter in the $[\mathrm{Mg} / \mathrm{Fe}]$ abundance ratio is fairly small: $\Delta([\mathrm{Mg} / \mathrm{Fe}])_{\mathrm{MAX}} \approx 0.6$ dex for all stars under consideration and $\Delta([\mathrm{Mg} / \mathrm{Fe}])_{\mathrm{MAX}} \approx 0.1$ dex for the M15 data set. In the metallicity regime below $[\mathrm{Fe} / \mathrm{H}] \lesssim-1.1$, the roughly consistent trend of $[\mathrm{Mg} / \mathrm{Fe}]$ abundance ratio is due in part to the production history for these elements: magnesium originates from hydrostatic burning in massive stars while iron is manufactured by massive star, core-collapse $\mathrm{SNe}$. If the short evolutionary lifetimes of these massive stars are taken into context with the abundance data, it would seem to indicate that the core-collapse $\mathrm{SNe}$ are rather ubiquitous events in the Galactic halo. Accordingly, the products that result from both stellar and explosive nucleosynthesis of massive stars should be well mixed in the interstellar and intercluster medium. The apparent downward trend in the $[\mathrm{Mg} / \mathrm{Fe}]$ ratio, in the metallicity region with $[\mathrm{Fe} / \mathrm{H}] \gtrsim-1.1$, is due to nucleosynthetic input from Type Ia $\mathrm{SNe}$, which produce much more iron in comparison to Type II events.

In a similar vein, the lower panel of Figure 12 plots $[\mathrm{Eu} / \mathrm{Fe}]$ as a function of $[\mathrm{Fe} / \mathrm{H}]$ and demonstrates that as the metallicity decreases, the spread in the $[\mathrm{Eu} / \mathrm{Fe}]$ abundance ratio increases enormously. ${ }^{15}$ By contrast, the scatter in the M15

15 Though the data sample of Figure 12 is compilation of several sources, the scatter in the $[\mathrm{Mg} / \mathrm{Fe}]$ and $[\mathrm{Eu} / \mathrm{Fe}]$ ratios duplicates that found by such large-scale surveys as, e.g., Barklem et al. (2005).
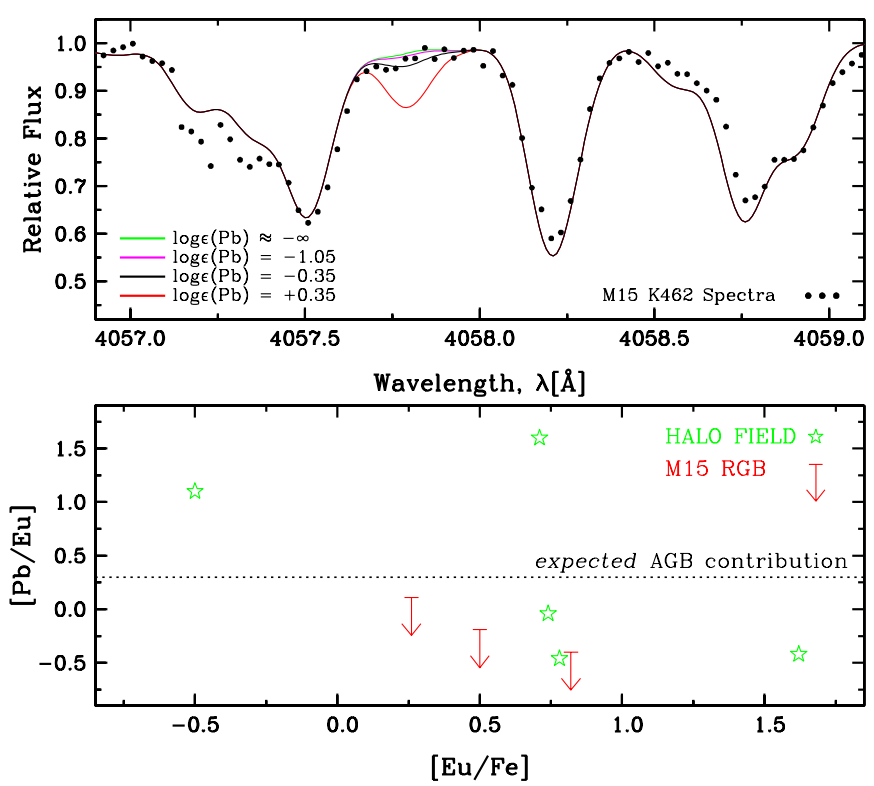

Figure 11. Upper panel: comparison of synthetic and observed spectra for the $\mathrm{Pb}$ I transition at $4057.8 \AA$ in the M15 K462 giant. Four incremental changes in abundance are shown for the specified $\mathrm{Pb}$ feature. It is only possible to establish an upper limit of approximately $\log \epsilon(\mathrm{Pb}) \approx-0.35$ for this star. A $\mathrm{CH}$ contaminant is present in the blue wing of the $\mathrm{Pb}$ transition and accounts for a definitive portion of the signal. Accordingly, it appears that the s-process element $\mathrm{Pb}$ is nominally detected in $\mathrm{K} 462$. Lower Panel: plot of $[\mathrm{Pb} / \mathrm{Eu}]$ as a function of $[\mathrm{Eu} / \mathrm{Fe}]$ for three M15 giants and five halo field stars. The empirically determined threshold ratio, which indicates the occurrence of AGB enrichment, is shown by the short-dashed line at $[\mathrm{Pb} / \mathrm{Eu}] \geqslant+0.3$ (this value is taken from Roederer et al. 2010; see the text for further details). Note that all of the M15 stars fall below this line (and, correspondingly, should lack main $s$-process material).

(A color version of this figure is available in the online journal.)
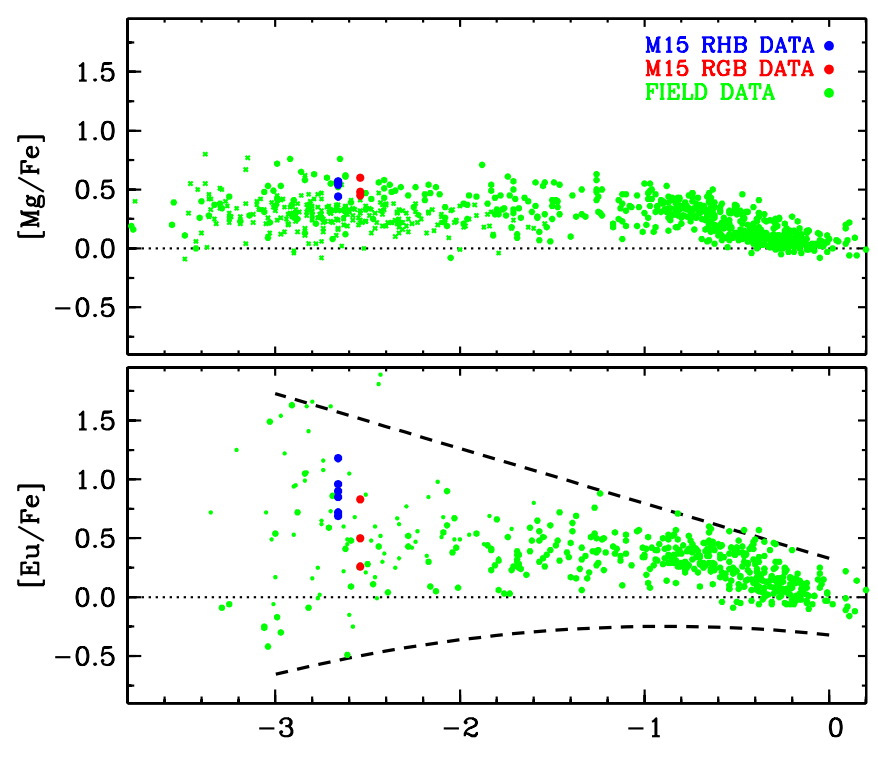

$[\mathrm{Fe} / \mathrm{H}]$

Figure 12. Comparison of the distribution $[(\mathrm{Mg}, \mathrm{Eu}) / \mathrm{Fe}]$ relative abundances as a function of metallicity for M15 targets (denoted by the blue and red circles) as well as for halo and disk stars (signified by the green, filled circles). Field star data have been taken from large sample surveys (see the text for further information). As per convention, the dotted lines represent the solar abundance ratios. In the lower panel, the two dashed black lines indicate the approximate ranges of the $[\mathrm{Eu} / \mathrm{Fe}]$ data. The spread in the $[\mathrm{Mg} / \mathrm{Fe}]$ values is smaller for $\mathrm{M} 15$ than that for the field. Yet for $[\mathrm{Eu} / \mathrm{Fe}]$, the M15 scatter is comparable to that of the field (for that particular metallicity).

(A color version of this figure is available in the online journal.) 
$[\mathrm{Eu} / \mathrm{Fe}]$ ratios is large and comparable to the spread of the halo field at that metallicity. Specifically in the metallicity interval $-2.7 \leqslant[\mathrm{Fe} / \mathrm{H}] \leqslant-2.2$, the scatter in the $[\mathrm{Eu} / \mathrm{Fe}]$ ratio is found to be $\sigma= \pm 0.27$ for the nine stars of the M15 sample and similarly for the 23 halo giants, the associated scatter is $\sigma= \pm 0.33$. This variation in the relative europium abundance ratio (as first detected by Gilroy et al. 1988 and later confirmed by others, e.g., Burris et al. 2000; Barklem et al. 2005) indicates an inhomogeneous production history for $\mathrm{Eu}$ and other corresponding $r$-process elements. These elements likely originate from lower mass $\mathrm{SNe}$ and their production is not correlated with that of the alpha elements (Cowan \& Thielemann 2004). Furthermore, it seems that nucleosynthetic events which generated the $r$-process elements were rare occurrences in the early Galaxy. As a consequence, these elements were not well mixed in the interstellar medium and intercluster medium (Sneden et al. 2009). Note that $r$-process enhancement seems to be a common feature of all GCs (e.g., Gratton et al. 2004). On the other hand, the scatter in select $r$-process element abundances, as found in M15, is not.

\section{SUMMARY}

A novel effort was undertaken to perform a homogenous abundance determination in both the RGB and RHB members of the M15 GC. The current investigation employed improved atomic data, stellar model atmospheres, and radiative transfer code. A resolute offset in the iron abundance between the RGB and RHB stars on the order of 0.1 dex was measured. Notwithstanding, the major findings of the analysis for both the RGB and RHB stellar groups include a definitive $r$-process enhancement, a significant spread in the abundances of the neutron-capture species (which appears to be astrophysical in nature), and an anti-correlation of light $n$-capture element abundance behavior with both barium $([\mathrm{Ba} / \mathrm{H}])$ and europium $([\mathrm{Eu} / \mathrm{H}])$. Accordingly, the last set of findings may offer proof of the operation of an LEPP-type mechanism within M15. To determine if these abundance behaviors are generally indicative of very metal-poor GCs, a comprehensive examination of the chemical composition of the analogous M92 cluster should be performed $([\mathrm{Fe} / \mathrm{H}] \sim-2.3$; Harris 1996; the literature contains relatively little information with regard to the $n$-capture abundances for this cluster).

To date, the presence of multiple stellar generations within the GC M15 has not been irrefutably established. In a series of papers, Carretta et al. (2009a, 2009c, 2010) offered compelling proof in the detection of light element anti-correlative behavior (Na-O) in numerous members of the M15 RGB. Lardo et al. (2011) did find a statistically significant spread in the SDSS photometric color index of $u-g$, but yet they were not able to demonstrate a clear and unambiguous correlation of $(u-g)$ with the $\mathrm{Na}$ abundances in the RGB of the M15 cluster (which would have provided additional evidence). To wit, recent investigations of M15 have revealed several atypical features including probable detection of an intermediate-mass black hole (van der Marel et al. 2002; though the result is under some dispute), observation of an ICM (Evans et al. 2003), detection of mass loss (Mészáros et al. 2008; Mészáros et al. 2009), identification of extreme horizontal branch and blue hook stars (Haurberg et al. 2010), and observation of an extended tidal tail (Chun et al. 2010). It would be worthwhile to examine these peculiar aspects of the GC in relation to the abundance results of M15. Further scrutiny is warranted in order to understand the star formation history and mixing timescale of the M15 protocluster environment.
We are deeply indebted to L. Koesterke for his extensive advice with regard to the modification of the MOOG code. We are grateful to the referee for several valuable suggestions. We also thank I. Roederer for helpful comments pertaining to drafts of the manuscript. The current effort has made use of the NASA Astrophysics Data System (ADS), the NIST Atomic Spectra Database (ASD), and the Vienna Atomic Line Database (VALD). Funding for this research has been generously provided by the National Science Foundation (grants AST 07-07447 to J.C. and AST 09-08978 to C.S.).

\section{APPENDIX}

\section{A.1. Alterations to the Line Transfer Code MOOG}

The essential approach to the solution of radiative transfer in the MOOG program has been altered with the employment of short characteristics and the application of an accelerated lambda iteration (ALI) scheme. Original development of the short characteristics (SC) methodology in the context of radiative transfer was done by Mihalas et al. (1978). Improvement of the SC approach in the explicit specification of the source function (at all grid points) was made by Olson \& Kunasz (1987) and Kunasz \& Auer (1988). As a supplemental reference, the current version of MOOG draws upon the concise treatment of Auer \& Paletou (1994). The implementation of the ALI technique within the framework of radiative transfer and stellar atmospheres was first done by Werner (1986) and subsequently refined by both, e.g., Rybicki \& Hummer (1991) and Hubeny (1992). The main SC and ALI prescription followed by the MOOG code is that from Koesterke et al. (2002, and references therein). Since the text below is a general description and pertains to the specific coding in MOOG, the reader should consult the aforementioned references as they contain significantly more information.

The primary modification to MOOG is the creation and incorporation of four new subroutines. The names and purposes of the new subroutines are as follows: AngWeight.f, which determines the Gaussian weights and integration points; Sourcefunc_scat_cont.f, which incorporates both a scattering and an absorption component to compute the source function and resultant flux for the continuum; Sourcefunc_scat_line.f, which incorporates both a scattering and an absorption component to compute the source function and resultant flux for the line; and, Cdcalc_JS.f, which calculates the final line depth via the emergent continuum and line fluxes. To accommodate these additions, several key subroutines were also revised. Further details and the publicly available MOOG code may be found at the Web site: http://www.as.utexas.edu/ chris/moog.html. Note that MOOG still retains the capacity to operate in pure absorption mode with the source function set simply to $S=\epsilon B$.

Ongoing and future improvements to the MOOG code include the incorporation of the Lee \& Kim (2004) formulation for Rayleigh scattering (for atomic hydrogen), the employment of a further discretization with regard to frequency, and the implementation of spherically symmetric geometry in the solution of radiative transfer.

\section{A.2. Contributions to the Continuous Opacity}

To remind the reader, in the visible spectral range, the two principal sources of opacity in stellar atmospheres are the bound-free absorption from the negative hydrogen ion $\left(\mathrm{H}^{-}\right)$and Rayleigh scattering from neutral atomic hydrogen. The standard 
expression (e.g., Gray 1976) for the $\mathrm{H}^{-}{ }_{\mathrm{BF}}$ absorption coefficient is

$$
\kappa \simeq 4.1458 \times 10^{-10} \alpha_{\mathrm{BF}} P_{e} \Theta^{5 / 2} 10^{0.754 \Theta},
$$

where $\alpha_{\mathrm{BF}}$ is the bound-free atomic absorption coefficient (which has frequency dependence), $P_{e}$ is the electron pressure, and $\Theta=5040 / T$ (note that Equation (A1) is per neutral hydrogen atom).

With regard to Rayleigh scattering, the scattering cross section of radiation with angular frequency $\omega$ incident upon a neutral $\mathrm{H}$ atom is given by the Kramers-Heisenberg formula in terms of atomic units as

$$
\frac{\sigma(\omega)}{\sigma_{T}}=\left(\frac{\omega}{\omega_{1}}\right)^{4}\left|A_{0}+A_{2}\left(\frac{\omega}{\omega_{1}}\right)^{2}+A_{4}\left(\frac{\omega}{\omega_{1}}\right)^{4}+\cdots\right|^{2},
$$

where $\sigma_{T}$ is the Thompson scattering cross section and $\omega_{1}$ is the angular frequency corresponding to the Lyman limit. Numerical calculations of the $A_{i}$ coefficients and the generation of an exact expression for Equation (A2) have been done by Dalgarno \& Williams (1962) and more recently by Lee \& Kim (2004).

The $\mathrm{H}^{-}{ }_{\mathrm{BF}}$ and Rayleigh scattering opacity contributions depend on temperature and metallicity (and, to some extent, on the surface gravity). Rayleigh scattering also has a $\lambda^{-4}$ dependence, and, as a consequence, it greatly influences blue wavelength transitions. For the majority of stars (such as dwarfs and subgiants), $\mathrm{H}^{-}{ }_{\mathrm{BF}}$ is the dominant opacity source in the visible spectral regime. However, for low-temperature, lowmetallicity giants, the Rayleigh scattering contribution becomes comparable to and even exceeds that from $\mathrm{H}^{-}{ }_{\mathrm{BF}}$ in the ultraviolet and blue visible wavelength regions. Therefore, to accurately determine the line intensity with the correct amount of flux and opacity contribution for all stellar types and over a wide spectral range, isotropic, coherent scattering must be considered.

\section{A.3. Form of the Radiative Transfer Equation and Implementation of the ALI Scheme}

The source function is then written as $S=(1-\epsilon) J+\epsilon B$ (where $\epsilon$ is the thermal coupling parameter, $J$ is the mean intensity, and $B$ is the Planck function). To commence with the formal solution of the radiation transfer equation, a fundamental assumption is made in that the source function is specified completely in terms of optical depth. After some mathematical manipulation, the RTE becomes

$$
\mu^{2} \frac{d^{2} j}{d \tau^{2}}=J-S
$$

where $\mu$ is the directional cosine and $\tau$ is the optical depth. Equation (A3) is an integro-differential equation (and subject to boundary conditions). To obtain the numerical solution of Equation (A3), a discretization in angle and optical depth is necessary. As a consequence, the solution is simplified and a Gaussian quadrature summation is done instead of an integration. Though it is eventually possible to evaluate Equation (A3) in a single step, the use of an iterative method to arrive at a solution is preferred as it is computationally faster than a straightforward approach. For the MOOG program, the ALI technique is employed with the application of a full acceleration. In the context of ALI scheme, the transfer equation takes the form of $J=\Lambda[S]$, where $\Lambda$ represents the matrix operator. Through the concept of preconditioning, ALI allows for the efficient, iterative solution of a (potentially) large system of linear equations. The main steps of the iterative cycle are the evaluation of $J=\Lambda[S]$, the computation of the $\Delta S$ quantity, and the corresponding adjustment to the source function.

\section{A.4. Short Characteristic Solution of Line Transfer}

From a general viewpoint, radiative transfer can be thought of as the propagation of photons along a ray on a two-dimensional grid. Note that the number of rays corresponds to the number of quadrature angles. Determination of radiation along a ray is done periodically at ray segments, or short characteristics. Essentially, SC start at a grid point and proceed along the ray until a cell boundary is met. At these cell boundaries, the intensity, opacity, and source function values are established. Then with the knowledge of the cell boundary intensities, the intensity at other non-grid points can be calculated.

Specifically with regard to MOOG, the intensity determination is a function of depths $(i)$ and angles $(j)$. It is performed for both an inward $(i-1)$ and an outward $(i+1)$ direction. Along the characteristic, the opacity quantity is assumed to be a linear function. In effect, the intensity for the ray can be expressed as

$$
I=I e^{-\Delta \tau(i)}+\int S(\tau) e^{-\tau} d \tau
$$

The optical depth step $\Delta \tau$ can be thought of as the path integral of the opacity along the characteristic (the entire, involved definition of the $\Delta \tau$ quantity is found in the Sourcefunc_scat_* subroutines). Now, the evaluation of Equation (A4) requires the interpolation of the source function. A linear interpolation is sufficient to satisfy the various boundary conditions. Interpolation over $[S(i), S(i \mp 1)]$ then entails

$$
\int S(\tau) e^{-\tau} d \tau=S(i) w_{1}(i)+S(i \mp 1) w_{2}(i) .
$$

The weights are given by the relations

$$
\begin{gathered}
w_{0}(i)=\left(e^{-\Delta \tau(i)}-1\right) / \Delta \tau(i), \\
w_{1}(i)=1+w_{0}(i), \\
w_{2}(i)=-e^{-\Delta \tau(i)}-w_{0}(i) .
\end{gathered}
$$

These weights are found by recursion. The use of linear interpolation does not generate significant error (as normally would occur) due to the optically thin nature of the boundary layer. The expression for the mean intensity, $J$, subsequently becomes

$$
J(i)=J(i)+0.5 w_{\mathrm{Gau}}(j) I,
$$

where the $w_{\text {Gau }}$ are the Gaussian quadrature weights (these are distinct from the weights of Equation (A5)). The summation over all depth points and angles(/rays) is necessarily performed. The SC formal solution of the transfer equation then proceeds in an iterative manner.

\section{REFERENCES}

Alonso, A., Arribas, S., \& Martínez-Roger, C. 1999, A\&AS, 140, 261 Anders, E., \& Grevesse, N. 1989, Geochim. Cosmochim. Acta, 53, 197 Andrievsky, S. M., Spite, M., Korotin, S. A., Spite, F., Bonifacio, P., Cayrel, R., Hill, V., \& François, P. 2007, A\&A, 464, 1081

Asplund, M. 2005, ARA\&A, 43, 481

Asplund, M., Grevesse, N., \& Sauval, A. J. 2005, in ASP Conf. Ser. 336, Cosmic Abundances as Records of Stellar Evolution and Nucleosynthesis, ed. T. G. Barnes III \& F. N. Bash (San Francisco, CA: ASP), 25 
Asplund, M., Grevesse, N., Sauval, A. J., \& Scott, P. 2009, ARA\&A, 47, 481

Auer, L. H., \& Paletou, F. 1994, A\&A, 285, 675

Baluja, K. L., \& Zeippen, C. J. 1988, J. Phys. B, 21, 1455

Barklem, P. S., \& O’Mara, B. J. 2000, MNRAS, 311, 535

Barklem, P. S., et al. 2005, A\&A, 439, 129

Bedin, L. R., Piotto, G., Anderson, J., Cassisi, S., King, I. R., Momany, Y., \& Carraro, G. 2004, ApJ, 605, L125

Bekki, K., Campbell, S. W., Lattanzio, J. C., \& Norris, J. E. 2007, MNRAS, 377, 335

Bernstein, R., Shectman, S. A., Gunnels, S. M., Mochnacki, S., \& Athey, A. E. 2003, Proc. SPIE, 4841, 1694

Biémont, E., Dutrieux, J.-F., Martin, I., \& Quinet, P. 1998, J. Phys. B, 31, 3321

Biémont, E., Garnir, H. P., Palmeri, P., Li, Z. S., \& Svanberg, S. 2000, MNRAS, 312, 116

Biémont, E., \& Godefroid, M. 1980, A\&A, 84, 361

Bizzarri, A., Huber, M. C. E., Noels, A., Grevesse, N., Bergeson, S. D., Tsekeris, P., \& Lawler, J. E. 1993, A\&A, 273, 707

Blackwell, D. E., Booth, A. J., Menon, S. L. R., \& Petford, A. D. 1986, MNRAS, 220, 289

Blackwell, D. E., Menon, S. L. R., \& Petford, A. D. 1982a, MNRAS, 201, 603

Blackwell, D. E., Menon, S. L. R., \& Petford, A. D. 1983, MNRAS, 204, 883

Blackwell, D. E., Menon, S. L. R., Petford, A. D., \& Shallis, M. J. 1982b, MNRAS, 201, 611

Blackwell, D. E., Petford, A. D., Shallis, M. J., \& Leggett, S. 1982c, MNRAS, 199,21

Blackwell-Whitehead, R., \& Bergemann, M. 2007, A\&A, 472, L43

Booth, A. J., Blackwell, D. E., Petford, A. D., \& Shallis, M. J. 1984, MNRAS, 208, 147

Brage, T., Wahlgren, G. M., Johansson, S. G., Leckrone, D. S., \& Proffitt, C. R. 1998, ApJ, 496, 1051

Buonanno, R., Buscema, G., Corsi, C. E., Iannicola, G., \& Fusi Pecci, F. 1983, A\&AS, 51, 83

Burris, D. L., Pilachowski, C. A., Armandroff, T. E., Sneden, C., Cowan, J. J., \& Roe, H. 2000, ApJ, 544, 302

Carbon, D. F., Romanishin, W., Langer, G. E., Butler, D., Kemper, E., Trefzger, C. F., Kraft, R. P., \& Suntzeff, N. B. 1982, ApJS, 49, 207

Carretta, E., Bragaglia, A., Gratton, R., D'Orazi, V., \& Lucatello, S. 2009a, A\&A, 508, 695

Carretta, E., Bragaglia, A., Gratton, R., \& Lucatello, S. 2009b, A\&A, 505, 139

Carretta, E., Bragaglia, A., Gratton, R., Lucatello, S., Bellazzini, M., \& D’Orazi, V. 2010, ApJ, 712, L21

Carretta, E., et al. 2009c, A\&A, 505, 117

Castelli, F., \& Kurucz, R. L. 2003, in IAU Symp. 210, Modelling of Stellar Atmospheres, ed. N. Piskunov, W. W. Weiss, \& D. F. Gray (Cambridge: Cambridge Univ. Press), 20

Cayrel, R., et al. 2004, A\&A, 416, 1117

Chang, T. N., \& Tang, X. 1990, JQSRT, 43, 207

Chun, S.-H., et al. 2010, AJ, 139, 606

Cohen, J. G., et al. 2004, ApJ, 612, 1107

Cowan, J. J., \& Thielemann, F.-K. 2004, Phys. Today, 57, 47

Cowan, J. J., et al. 2005, ApJ, 627, 238

Cudworth, K. M. 1976, AJ, 81, 519

Dalgarno, A., \& Williams, D. A. 1962, ApJ, 136, 690

Den Hartog, E. A., Lawler, J. E., Sneden, C., \& Cowan, J. J. 2003, ApJS, 148 , 543

Den Hartog, E. A., Lawler, J. E., Sneden, C., \& Cowan, J. J. 2006, ApJS, 167, 292

Doerr, A., Kock, M., Kwiatkowski, M., \& Werner, K. 1985, J. Quant. Spectrosc. Radiat. Transfer, 33, 55

Dotter, A., Chaboyer, B., Jevremović, D., Kostov, V., Baron, E., \& Ferguson, J. W. 2008, ApJS, 178, 89

Evans, A., Stickel, M., van Loon, J. T., Eyres, S. P. S., Hopwood, M. E. L., \& Penny, A. J. 2003, A\&A, 408, L9

Farouqi, K., Kratz, K.-L., Mashonkina, L. I., Pfeiffer, B., Cowan, J. J., Thielemann, F.-K., \& Truran, J. W. 2009, ApJ, 694, L49

Fischer, C. 2004, MCHF/MCDHF Collection, Version 2, http://physics.nist.gov/ mchf

Fischer, C., \& Tachiev, G. 2003, MCHF/MCDHF Collection, Version 2, http://physics.nist.gov/mchf

Fitzpatrick, M. J., \& Sneden, C. 1987, BAAS, 19, 1129

François, P., et al. 2007, A\&A, 476, 935

Fröhlich, C., Martínez-Pinedo, G., Liebendörfer, M., Thielemann, F.-K., Bravo, E., Hix, W. R., Langanke, K., \& Zinner, N. T. 2006, Phys. Rev. Lett., 96, 142502

Fuhr, J. R., \& Wiese, W. L. 1998, in CRC Handbook of Chemistry and Physics, 79th Edition, ed. D. R. Lide (Boca Raton, FL: CRC Press)

Fuhr, J. R., \& Wiese, W. L. 2006, J. Phys. Chem. Ref. Data, 35, 1669
Fulbright, J. P. 2000, AJ, 120, 1841

Garz, T. 1973, A\&A, 26, 471

Gilroy, K. K., Sneden, C., Pilachowski, C. A., \& Cowan, J. J. 1988, ApJ, 327, 298

Gratton, R. G., \& Sneden, C. 1994, A\&A, 287, 927

Gratton, R., Sneden, C., \& Carretta, E. 2004, ARA\&A, 42, 385

Gray, D. F. 1976, The Observation and Analysis of Stellar Photospheres (New York: Wiley)

Grevesse, N., Asplund, M., Sauval, A. J., \& Scott, P. 2010, Ap\&SS, 328, 179

Gustafsson, B., Edvardsson, B., Eriksson, K., Jørgensen, U. G., Nordlund, Å., \& Plez, B. 2008, A\&A, 486, 951

Hannaford, P., Lowe, R. M., Grevesse, N., Biemont, E., \& Whaling, W. 1982, ApJ, 261, 736

Han, S.-I., Lee, Y.-W., Joo, S.-J., Sohn, S. T., Yoon, S.-J., Kim, H.-S., \& Lee, J.-W. 2009, ApJ, 707, L190

Harris, W. E. 1996, AJ, 112, 1487

Haurberg, N. C., Lubell, G. M. G., Cohn, H. N., Lugger, P. M., Anderson, J., Cool, A. M., \& Serenelli, A. M. 2010, ApJ, 722, 158

Helfer, H. L., Wallerstein, G., \& Greenstein, J. L. 1959, ApJ, 129, 700

Honda, S., Aoki, W., Ishimaru, Y., Wanajo, S., \& Ryan, S. G. 2006, ApJ, 643, 1180

Hubeny, I. 1992, in The Atmospheres of Early-Type Stars, ed. U. Heber \& C. S. Jeffery (Lecture Notes in Physics, Vol. 401; Berlin: Springer), 377

Huber, M. C. E., \& Sandeman, R. J. 1980, A\&A, 86, 95

Ivans, I. I., Simmerer, J., Sneden, C., Lawler, J. E., Cowan, J. J., Gallino, R., \& Bisterzo, S. 2006, ApJ, 645, 613

Johnson, J. A. 2002, ApJS, 139, 219

Karamatskos, N., Michalak, R., Zimmermann, P., Kroll, S., \& Kock, M. 1986, Z. Phys. D, 3, 391

Kerkhoff, H., Schmidt, M., \& Zimmermann, P. 1980, Z. Phys., 298, 249

King, J. R., Stephens, A., Boesgaard, A. M., \& Deliyannis, C. 1998, AJ, 115 , 666

Koesterke, L., Hamann, W.-R., \& Gräfener, G. 2002, A\&A, 384, 562

Korn, A. J., Grundahl, F., Richard, O., Mashonkina, L., Barklem, P. S., Collet, R., Gustafsson, B., \& Piskunov, N. 2007, ApJ, 671, 402

Köstlin, H. 1964, Zeitschrift fur Physik, 178, 200

Kraft, R. P., \& Ivans, I. I. 2003, PASP, 115, 143

Kratz, K.-L., Farouqi, K., Pfeiffer, B., Truran, J. W., Sneden, C., \& Cowan, J. J. 2007, ApJ, 662, 39

Kunasz, P., \& Auer, L. H. 1988, J. Quant. Spectrosc. Radiat. Transfer, 39, 67

Kupka, F., Ryabchikova, T. A., Piskunov, N. E., Stempels, H. C., \& Weiss, W. W. 2000, Balt. Astron., 9, 590

Kurucz, R. L. 1993, Kurucz CD-ROM 13, ATLAS9 Stellar Atmosphere Programs and $2 \mathrm{~km} \mathrm{~s}^{-1}$ Grid (Cambridge: SAO)

Kurucz, R. L. 2005, Mem. Soc. Astron. Ital., 8, 14

Kustner, F. 1921, Veroeffentlichungen Astron. Inst. Univ. Bonn, 15,

Lai, D. K., Bolte, M., Johnson, J. A., Lucatello, S., Heger, A., \& Woosley, S. E. 2008, ApJ, 681, 1524

Lai, D. K., Johnson, J. A., Bolte, M., \& Lucatello, S. 2007, ApJ, 667, 1185

Lardo, C., Bellazzini, M., Pancino, E., Carretta, E., Bragaglia, A., \& Dalessandro, E. 2011, A\&A, 525, A114

Lawler, J. E., Bonvallet, G., \& Sneden, C. 2001a, ApJ, 556, 452

Lawler, J. E., \& Dakin, J. T. 1989, J. Opt. Soc. Am. B, 6, 1457

Lawler, J. E., Den Hartog, E. A., Labby, Z. E., Sneden, C., Cowan, J. J., \& Ivans, I. I. 2007, ApJS, 169, 12

Lawler, J. E., Den Hartog, E. A., Sneden, C., \& Cowan, J. J. 2006, ApJS, 162 , 227

Lawler, J. E., Sneden, C., \& Cowan, J. J. 2004, ApJ, 604, 850

Lawler, J. E., Sneden, C., Cowan, J. J., Ivans, I. I., \& Den Hartog, E. A 2009, ApJS, 182, 51

Lawler, J. E., Sneden, C., Cowan, J. J., Wyart, J.-F., Ivans, I. I., Sobeck, J. S., Stockett, M. H., \& Den Hartog, E. A. 2008, ApJ, 178, 71

Lawler, J. E., Wickliffe, M. E., Cowley, C. R., \& Sneden, C. 2001b, ApJS, 137, 341

Lawler, J. E., Wickliffe, M. E., den Hartog, E. A., \& Sneden, C. 2001c, ApJ, 563, 1075

Lee, H.-W., \& Kim, H. I. 2004, MNRAS, 347, 802

Li, R., Chatelain, R., Holt, R. A., Rehse, S. J., Rosner, S. D., \& Scholl, T. J. 2007, Phys. Scr., 76, 577 (Li07)

Marigo, P., Girardi, L., Bressan, A., Groenewegen, M. A. T., Silva, L., \& Granato, G. L. 2008, A\&A, 482, 883

Marino, A. F., Milone, A. P., Piotto, G., Villanova, S., Bedin, L. R., Bellini, A., \& Renzini, A. 2009, A\&A, 505, 1099

Mendoza, C., Eissner, W., Le Dourneuf, M., \& Zeippen, C. J. 1995, J. Phys. B, 28, 3485

Mészáros, S., Avrett, E. H., \& Dupree, A. K. 2009, AJ, 138, 615

Mészáros, S., Dupree, A. K., \& Szentgyorgyi, A. 2008, AJ, 135, 1117 
Mihalas, D., Auer, L. H., \& Mihalas, B. R. 1978, ApJ, 220, 1001

Nahar, S. N., \& Pradhan, A. K. 1993, J. Phys. B, 26, 1109

Nilsson, H., Ljung, G., Lundberg, H., \& Nielsen, K. E. 2006, A\&A, 445, 1165

Nilsson, H., Zhang, Z. G., Lundberg, H., Johansson, S., \& Nordström, B. 2002, A\&A, 382, 368

Nitz, D. E., Kunau, A. E., Wilson, K. L., \& Lentz, L. R. 1999, ApJS, 122, 557

O’Brian, T. R., \& Lawler, J. E. 1991, Phys. Rev. A, 44, 7134

O’Brian, T. R., Wickliffe, M. E., Lawler, J. E., Whaling, W., \& Brault, J. W. 1991, J. Opt. Soc. Am. B, 8, 1185

Olson, G. L., \& Kunasz, P. B. 1987, J. Quant. Spectrosc. Radiat. Transfer, 38, 325

Otsuki, K., Honda, S., Aoki, W., Kajino, T., \& Mathews, G. J. 2006, ApJ, 641, L117

Palmeri, P., Quinet, P., Wyart, J.-F., \& Biémont, E. 2000, Phys. Scr., 61, 323

Pfeiffer, B., Ott, U., \& Kratz, K.-L. 2001, Nucl. Phys. A, 688, 575

Pickering, J. C., Thorne, A. P., \& Perez, R. 2001, ApJS, 132, 403

Pinnington, E. H., Rieger, G., \& Kernahan, J. A. 1997, Phys. Rev. A, 56, 2421

Piotto, G., et al. 2007, ApJ, 661, L53

Plez, B., \& Cohen, J. G. 2005, A\&A, 434, 1117

Preston, G. W., Sneden, C., Thompson, I. B., Shectman, S. A., \& Burley, G. S. 2006, AJ, 132, 85

Qian, Y.-Z., \& Wasserburg, G. J. 2000, Phys. Rep., 333, 7

Qian, Y.-Z., \& Wasserburg, G. J. 2002, ApJ, 567, 515

Quinet, P., Palmeri, P., Biémont, É., Jorissen, A., van Eck, S., Svanberg, S., Xu, H. L., \& Plez, B. 2006, A\&A, 448, 1207

Reddy, B. E., Lambert, D. L., \& Allende Prieto, C. 2006, MNRAS, 367, 1329

Reddy, B. E., Tomkin, J., Lambert, D. L., \& Allende Prieto, C. 2003, MNRAS, 340,304

Roederer, I. U., Cowan, J. J., Karakas, A. I., Kratz, K.-L., Lugaro, M., Simmerer, J., Farouqi, K., \& Sneden, C. 2010, ApJ, 724, 975

Rybicki, G. B., \& Hummer, D. G. 1991, A\&A, 245, 171

Scott, P., Asplund, M., Grevesse, N., \& Sauval, A. J. 2009, ApJ, 691, L119

Simmerer, J., Sneden, C., Cowan, J. J., Collier, J., Woolf, V. M., \& Lawler, J. E. 2004, ApJ, 617, 1091

Skrutskie, M. F., et al. 2006, AJ, 131, 1163
Sneden, C. 1973, ApJ, 184, 839

Sneden, C., Cowan, J. J., \& Gallino, R. 2008, ARA\&A, 46, 241

Sneden, C., Johnson, J., Kraft, R. P., Smith, G. H., Cowan, J. J., \& Bolte, M. S. 2000a, ApJ, 536, L85

Sneden, C., Kraft, R. P., Prosser, C. F., \& Langer, G. E. 1991, AJ, 102, 2001

Sneden, C., Kraft, R. P., Shetrone, M. D., Smith, G. H., Langer, G. E., \& Prosser, C. F. 1997, AJ, 114, 1964

Sneden, C., Lawler, J. E., Cowan, J. J., Ivans, I. I., \& Den Hartog, E. A 2009, ApJS, 182, 80

Sneden, C., Pilachowski, C. A., \& Kraft, R. P. 2000b, AJ, 120, 1351

Sneden, C., et al. 2003, ApJ, 591, 936

Sobeck, J. S., Lawler, J. E., \& Sneden, C. 2007, ApJ, 667, 1267

Sweigart, A. V. 1976, Phys. Today, 29, 25

Thielemann, F.-K., et al. 2001, Prog. Part. Nucl. Phys., 46, 5

Thompson, I. B., et al. 2008, ApJ, 677, 556

Travaglio, C., Gallino, R., Arnone, E., Cowan, J., Jordan, F., \& Sneden, C. 2004, ApJ, 601, 864

van der Marel, R. P., Gerssen, J., Guhathakurta, P., Peterson, R. C., \& Gebhardt, K. 2002, AJ, 124, 3255

Vogt, S. S., et al. 1994, Proc. SPIE, 2198, 362

Volz, U., Majerus, M., Liebel, H., Schmitt, A., \& Schmoranzer, H. 1996, Phys. Rev. Lett., 76, 2862

Wasserburg, G. J., \& Qian, Y.-Z. 2000, ApJ, 529, L21

Werner, K. 1986, A\&A, 161, 177

Westin, J., Sneden, C., Gustafsson, B., \& Cowan, J. J. 2000, ApJ, 530, 783

Whaling, W., Hannaford, P., Lowe, R. M., Biemont, E., \& Grevesse, N. 1985 A\&A, 153, 109

Wickliffe, M. E., \& Lawler, J. E. 1997, J. Opt. Soc. Am. B, 14, 737

Wickliffe, M. E., Lawler, J. E., \& Nave, G. 2000, J. Quant. Spectrosc. Radiat. Transfer, 66, 363

Wujec, T., \& Musielok, J. 1986, J. Quant. Spectrosc. Radiat. Transfer, 36, 7

Yanny, B., Guhathakurta, P., Bahcall, J. N., \& Schneider, D. P. 1994, AJ, 107, 1745

Youssef, N. H., \& Khalil, N. M. 1988, A\&A, 203, 378

Zinn, R., \& West, M. J. 1984, ApJS, 55, 45 